\title{
Measuring bar strength using Fourier analysis of galaxy images
}

\author{
C. Garcia-Gómez ${ }^{1}$, E. Athanassoula ${ }^{2}$, C. Barberà ${ }^{1}$, and A. Bosma ${ }^{2}$ \\ 1 DEIM, Campus Sescelades, Avd. dels Països Catalans 26, 43007 Tarragona, Spain \\ e-mail: carlos . barbera@urv.cat \\ ${ }^{2}$ LAM (Laboratoire d'Astrophysique de Marseille), CNRS, Aix Marseille Université, UMR 7326, 13388 Marseille, France
}

Received 1 May 2016 / Accepted 13 February 2017

\begin{abstract}
We present two methods for bar strength measurement on disc galaxies based on a two-dimensional Fourier analysis of their images. Our methods are able to recover the whole spiral structure of disc galaxies and are very robust when comparing the results from images obtained with different filters. In particular, these methods are capable of detecting small bars regardless of the filter used to obtain the galaxy image. Our relative measurements of bar strength show a good agreement with different bar indicators used in the literature. We use our methods to determine the general morphologies indicated in the Third Reference Catalog of Bright Galaxies (RC3 catalogue) classified as non-barred (SA), mildly barred (SAB), or barred (SB) galaxies. Our numerical indicators are in good agreement with the three galaxy populations estimated visually.
\end{abstract}

Key words. galaxies: structure - galaxies: spiral

\section{Introduction}

Most disc galaxies show bar components when studied in the near-infrared (NIR; Eskridge et al. 2000; Knapen et al. 2000; Grosbol et al. 2004; Marinova \& Jogee 2007; Menéndez-Delmestre et al. 2007). The strength of the detected bar, however, varies within a very wide range, from very strong and long bars to small short bars or more massive ovals.

Intuitively, the difference between a weak and strong bar is clear, but there is no unique definition of bar strength. Thus, a bar is called strong if it includes a large portion of light (or mass) of a galaxy, but sometimes longitude and thickness of the bar is also considered. But, is a very thin short bar stronger or weaker than a long massive oval? The answer is not unique and depends on the definition used.

Different attempts to measure the bar strength can be found in the literature. Elmegreen \& Elmegreen (1985) and Regan \& Elmegreen (1997) used the ratio between the peak surface brightness in the bar to the minimum surface brightness in the interbar region. Martin (1995), Martinet \& Friedli (1997), and Chapelon et al. (1999) used the axis ratio and bar length. Rozas et al. (1998) used the ratio of the flux inside the bar to the flux outside excluding the bulge. Aguerri et al. (1998) and Aguerri et al. (2000) used the ratio between the amplitudes of the Fourier $m=2$ and $m=0$ components. Seigar \& James (1998) used a parameter called the "equivalent angle" defined as the angle subtended at the centre of the galaxy by a sector of the underlying disc and bulge that emits as much light as the bar component within the same radial limits. Abraham \& Merrifield (2000) introduced the parameter

$f_{\mathrm{bar}}=\frac{2}{\pi}\left[\arctan \left(\frac{b}{a}\right)_{\mathrm{bar}}^{-1 / 2}-\arctan \left(\frac{b}{a}\right)_{\mathrm{bar}}^{+1 / 2}\right]$,

where $(b / a)_{\text {bar }}$ is the intrinsic axial ratio of the bar that is obtained from the deprojected image or calculated from its apparent axial ratio and inclination of the galaxy (e.g. Abraham et al. 1999). This parameter is closely related to a morphologist's subjective notion of bar strength and maps the bar strength into a closed interval from zero to unity. A nonbarred galaxy has a value of $f_{\text {bar }}=0$, while an ideal galaxy with a bar of infinite strength has a value of $f_{\mathrm{bar}}=1$. Galaxies with a value of $f_{\text {bar }}>0.11$ correspond to systems in which $(b / a)^{2}<0.5$ and should be classified as barred, as shown in Abraham et al. (1999). This parameter was used by Whyte et al. (2002) to study the bar distribution in the Ohio State University Bright Spiral Galaxy Survey (hereafter OSU) sample of bright galaxies Eskridge et al. (2000) and showed some evidence of a bi-modality in the distribution that would suggest that barred and unbarred galaxies are not the extreme of a single distribution. Aguerri (1999) used the parameter $\epsilon_{b}=10(1-b / a)$ as a measure of the bar strength and showed that there is a strong correlation between bar strength and star formation activity; Shlosman et al. (2000) also used this parameter to show that in active galaxies there is a deficiency of "thin bars" (i.e. bars with high ellipticity). Marinova \& Jogee (2007) have recently used this parameter to study the distribution of barred galaxies in the OSU sample. Using this parameter, these authors do not find evidence of a bimodal distribution, and note that bar fraction and bar strength do not seem to depend on Hubble type. They also note that a significant portion of unbarred galaxies in the Third Reference Cata$\log$ of Bright Galaxies (RC3 catalogue) by de Vaucouleurs et al. (1991) turn out to be barred when studied in $H$ band images. This higher portion of barred galaxies in the infrared had already been referred to by Eskridge et al. (2000) on visual inspection.

Using numerical simulations of the dynamics of disc galaxies, Combes \& Sanders (1981) introduced the bar torque as measured by the $Q_{\mathrm{b}}$ parameter, which is a measure of the relative amplitude of the second harmonic over the axisymmetric force. Buta \& Block (2001) and Laurikainen \& Salo (2002) used this bar torque $Q_{\mathrm{b}}$ as a measure of bar strength. This parameter is defined as the maximum value of the ratio of the tangential force to the mean axisymmetric radial force, i.e. it is a 
local criterion. When using images in the $K$ band, Block et al. (2001) noted that this parameter correlates only weakly with the optical bar type as listed in the Revised Shapley Ames by Sandage \& Tammann (1981) and de Vaucouleurs (RC3) catalogues. This could be because some bars with strong bulges have their azimuthal forces diluted by the average radial force exerted by strong bulges. Block et al. (2002), Laurikainen et al. (2004), and Buta et al. (2004) have calculated this parameter for the galaxy images of the OSU sample. This parameter correlates well with deprojected bar ellipticities, even though the calculation of this parameter requires some model assumptions on the vertical density distribution of the discs (Laurikainen \& Salo 2002). Such a correlation is to be expected as the shape of the bar is related to the shape of the orbits supporting it and these should depend on the global force field. Buta et al. (2004) also showed that the $Q_{\mathrm{b}}$ parameter correlates well with the $f_{\mathrm{bar}}$ parameter derived by Whyte et al. (2002) for the OSU sample.

Nevertheless, the $Q_{\mathrm{b}}$ parameter can be influenced by the torques exerted by spiral arms. Buta et al. (2003) introduced a method that separates the influence of the spiral arms from that of the bar. This methodology was based on the previous analysis of some barred galaxies by Ohta et al. (1990). This method uses the assumption that the distribution of the Fourier amplitudes as a function of radius are symmetric for each component. The main component is thus identified in the even harmonics and subtracted from the galaxy image, so that the bar strength can be calculated separately from that of the arm. In this case, however, strong symmetric arms can couple nonlinearly with the bar component and a direct substraction of the arm component can give an unreliable value of $Q_{\mathrm{b}}$. Buta et al. (2005) applied this method to the galaxy images in the OSU sample and found that the distribution of bar strengths declines smoothly with increasing $Q_{\mathrm{b}}$, where more than $40 \%$ of the sample have $Q_{\mathrm{b}} \leq 0.1$. The $Q_{\mathrm{b}}$ parameter can also be influenced by the existence of a classical bulge. For this reason, Durbala et al. (2009) first subtract this bulge contribution from the image before calculating the numerical value of the $Q_{\mathrm{b}}$ parameter.

Finally, Speltincx et al. (2008) applied this parameter to the OSU sample to compare the bar strengths in the $B$ and $H$ filters and concluded that the ratio of bar strengths in the two filters is on average $Q_{B / H}=1.25$ and that this is mainly due to the reduced bulge dilution of the radial forces in the $B$ band. They showed also that this parameter could be used with confidence to quantify bar strength at high redshifts.

Other bar strength definitions can be obtained from reasonable combinations of the bar strength attributes, i.e. its length, axial ratio, and mass. In some simplifying cases, only one of these attributes is used, which leads to clear-cut measuring systems, which, nevertheless, do not take into account all the bar properties. This is not, however, necessarily a disadvantage, since the right mix of attributes in any strength formula is unknown. In fact, there is no right or best strength criterion. Some criteria are more adequate than others for a given application, while other criteria may be more suitable for another particular case. Here we introduce and test some new criteria for the bar strength, all of which take the global (as opposed to local) strength of the bar into account. They are all based on the Fourier analysis of galaxy images and are introduced in the following sections.

This paper is organised as follows: Sect. 2 gives a general introduction to the two-dimensional Fourier analysis technique and, as an example, applies it to a disc galaxy with a welldeveloped global bi-symmetric pattern. In Sect. 3 we present the sample and the new measures of bar strength. Section 4 applies this technique to model barred galaxies and to some representative examples of galaxies. Section 5 compares these measures between them and discusses the effect of wavelength on the bar strength. In Sect. 6 we compare our values to those previously used and in Sect. 7 we make a global comparison of the values measured by our indicators with the classification as non-barred (SA), mildly barred (SAB), or barred (SB) galaxies according to the RC3 catalogue. We conclude in Sect. 8.

\section{Fourier analysis technique}

The two-dimensional fast Fourier transform (FFT) method, which analyses the spiral structure of disc galaxies, was first introduced by Considère \& Athanassoula (1982) for the HII region distribution and, independently, by Iye et al. (1982) for a photometric image of the galaxy NGC 4254 Considère \& Athanassoula (1988) used it for a sample of images of bright spiral galaxies. It was later fully developed by García-Gómez \& Athanassoula (1991), who introduced the possibility of dealing with the whole complexity of Fourier spectra, separating all the signals present in each spectrum. In this paper, we apply this method to galaxy images and, in this section, start by summarizing the main points. More information can be found in Barberà et al. (2004), and García-Gómez et al. (1991).

We use the galaxy images in the bright galaxy sample of Frei et al. (1996) and in the OSU sample. As we are only interested in the relative intensities, we use the uncalibrated images, first removing foreground stars and subtracting the sky background. We then centre the images on the pixel with the highest density. The next step is to deproject the galaxy image $I_{p}(r, \theta)$ using the position angles (hereafter PA) and inclination angles (hereafter IA) of each galaxy to obtain the light distribution of the galaxy on its equatorial plane $I(r, \theta)$. The step of deprojecting galaxy images is a crucial phase of the whole process because a bad deprojection contributes to the Fourier spectra with spurious signals. The deprojection of the galaxy images has been treated in detail separately for the Frei and OSU sample in the papers by Barberà et al. (2004), and García-Gómez et al. (1991), respectively, but we briefly describe the process for the sake of completeness. Basically, the Fourier deprojection method aims to minimise the relative power of the component associated with an oval distortion. Any oval component gives a strong signal in the bysymmetric component (i.e. a symmetry after a rotation of $\pi$ degrees). If we choose a bad pair of deprojecting angles (PA, IA) the galaxy disc looks oval and, hence, we get a stronger bi-symmetric signal in the Fourier spectrum. Thus, we can obtain a good guess of the deprojection angles by comparing the power in this component over the rest of the components of the spectrum.

Given the deprojected image of the galaxy $I(u, \theta)$, where $u=\ln (r)$, we calculate the two-dimensional Fourier transform defined as

$A(p, m)=\int_{u_{\min }}^{u_{\max }} \int_{0}^{2 \pi} I(u, \theta) \mathrm{e}^{i(p u+m \theta)} \mathrm{d} \theta \mathrm{d} u$.

The Fourier transform is a very convenient tool for the analysis of the spiral structure as we decompose the galaxy image using the orthogonal basis of logarithmic spirals. Spiral arms are known to follow this function approximately (Danver 1942; Kennicutt 1981; Ma 2001). In Eq. (2), $p$ corresponds to the radial frequency and $m$ to the azimuthal frequency. While $p$ is a real variable, $m$ only takes integer values. The spectra obtained with $m=1$ correspond to one-armed spirals, or asymmetries 
within the galaxy image. The $m=2$ spectra correspond to bisymmetric signals, i.e. signals with a periodicity of $\pi$ radians. These signals are associated with the common two-armed spirals, which look the same after a rotation by $\pi$ radians around the galaxy centre, or to a bar. Similarly, the $m=3$ spectrum are associated with signals corresponding to three-armed spirals and so on for the spectrum of $m=4$ and higher azimuthal frequencies. The radial frequency $p$ is related to the pitch angle $i$ of the spiral arm via the relation

$p=-\frac{m}{\tan (i)}$

In order to obtain a good resolution in the frequency space, we fix the range of radial frequencies, $\left(-p_{\max }, p_{\max }\right)$, by setting $p_{\max }=128$ and with a rate of sampling of $\delta p=0.125$, giving a total of $N=2^{11}$ samples in the Fourier transform, which is very adequate for our purposes. The value of $\delta u$ is fixed to the value of the maximum radial frequency through the expression

$\delta u=\frac{2 \pi}{N \delta p}=\frac{\pi}{p_{\max }}$.

Then, the range of radial frequencies is linked to the range of $u$ values $\left(-u_{\max }, u_{\max }\right)$ by the relation

$u_{\max }=\frac{N \delta u}{2}=\frac{\pi N}{2 p_{\max }}$.

In order to avoid aliasing (Gibbs phenomenon) we taper the values in the central part of the galaxy by the function

$F=\left\{\begin{aligned} \sin ^{2}\left(\frac{\pi}{2} \frac{r}{r_{\min }}\right): & r<r_{\min } \\ 1: & r \geq r_{\min } .\end{aligned}\right.$

Here the length $r_{\min }$ depends on the structure of the galaxy and is obtained for each galaxy image separately after visual inspection. This $r_{\min }$ was selected as a fraction of the radius at 25th magnitude $(R 25)$ of each galaxy. When this selection was made we calculated the galaxy spectra and ensured that the spectra of all $m$ values were not dominated by the signal of the central bulge. Bulges are not perfectly round and symmetric and, if the value of the $r_{\min }$ is selected too small, bulge residuals dominate all the spectra through higher harmonics. Thus, an asymmetric bulge gives strong signals in the $m=1$, while a symmetric bulge entirely dominates the $m=2$ component. These signals also dominate the corresponding associated higher harmonics. If such very bright structures are not tapered out, they can dominate the Fourier transform and hide the contribution of the remaining spiral components. This procedure is described in Fig. 1, where we show the effect of the selection of the value of $r_{\min }$ on the spectra of the galaxy NGC 4303 . When using a value that is $20 \%$ lower than the value actually selected, the $m=1$ spectrum is dominated by a strong signal in the central part, centred around values of $p=0$. On the other hand, when using the selected value of $r_{\min }$ the spectra are dominated by the signals in the $m=2$ spectrum, i.e. the central oval of this galaxy and the arms. This signal does not change appreciably when using a value of $r_{\min }$ $20 \%$ higher than the value actually used.

Following the above described procedure, we obtain a series of spectra for each galaxy image: one for each value of the azimuthal frequency $m$. Each of these spectra is combination of signals corresponding to spirals with the same multiplicity but with different pitch angles, each associated with different values of the radial frequency $p$.

In Fig. 2, we show the modulus of the $m=2$ Fourier spectrum of the galaxy NGC 4535 obtained from an $r$ band image,

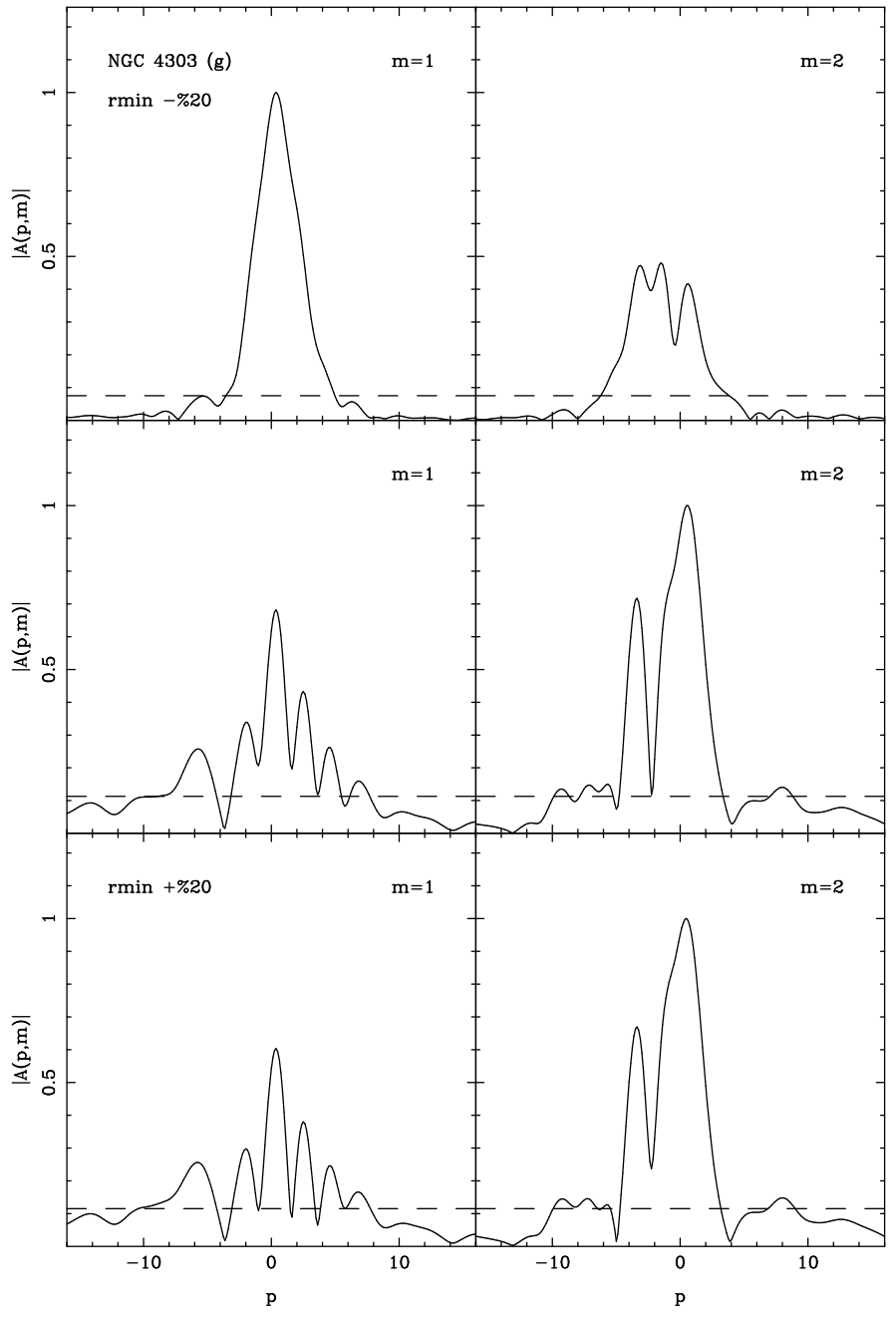

Fig. 1. Upper panel: modulus of the $m=1$ and $m=2$ Fourier spectra obtained from a $g$ band image of the galaxy NGC 4303 using a $r_{\min }$ value lower by a $20 \%$. Middle panel: same spectra obtained the right value of $r_{\min }$. Lower panel: spectra obtained using a value of $r_{\min } 20 \%$ higher.

and we plot normalised amplitudes for the spectra. We are only interested in the relative amplitudes of the signals and this means that the galaxy images do not need to be calibrated when comparing the signals of each galaxy. As can be seen in Fig. 2, the different spectra of each galaxy can be very complex. This means that many different signals contribute to each spectrum and, in order to recover the galaxy image properly, we need to unravel this complexity. We do so by fitting the modulus of each spectrum by a sum of Gaussians as follows:

$|A(p, m)|=\sum_{j=1}^{N_{\mathrm{g}}} C_{j} \exp \left(-\frac{\left(p-p_{j}\right)^{2}}{2 \sigma_{j}^{2}}\right)$.

In this relation, $p_{j}$ represents the central frequency of the Gaussian, $\sigma_{j}$ its dispersion, and $C_{j}$ its amplitude. The number of Gaussians used in each fit, $N_{\mathrm{g}}$, depends on the complexity of the spectrum and thus varies from spectrum to spectrum and from galaxy to galaxy. In the upper panel of Fig. 2 we show, as a typical case, the result of this fitting process to the $m=2$ spectrum of the image in the $r$ band of the galaxy NGC 4535 . We have used a total of five Gaussians for this particular fit and the fit obtained is excellent; the difference between the modulus 


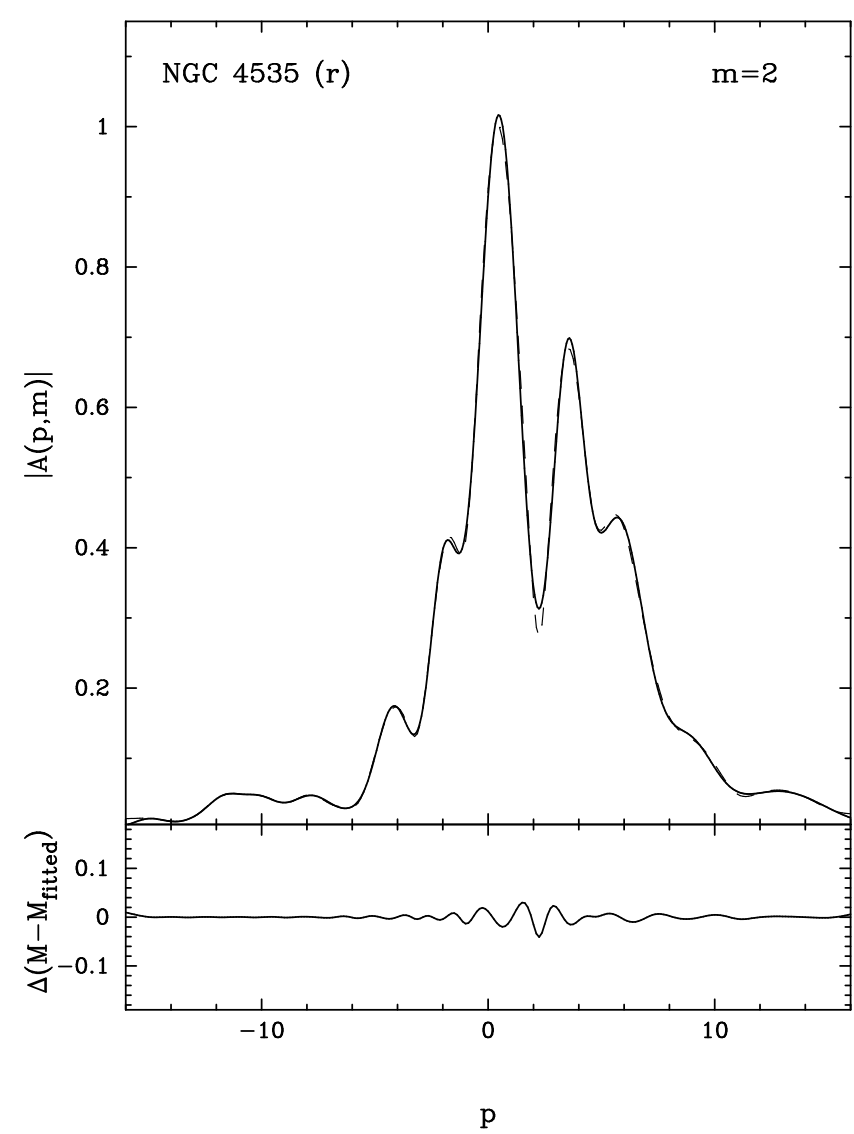

Fig. 2. Upper panel: modulus of the $m=2$ Fourier spectrum obtained from an $r$ band image of the galaxy NGC 4535. This modulus has considerable substructure, due to the presence of many components. We superimpose using a dotted line the Gaussian fit, obtained as described in the text. Lower panel: difference between the value of the $m=2$ modulus and the Gaussian fit. This fit, whose quality is typical for our sample, reproduces very well the $m=2$ modulus, in spite of the latter's complexity.

and the fit is hardly visible over most of the range of the radial frequencies $p$. The small difference between the modulus of the spectrum and the Gaussian fit can be measured in the lower panel of Fig. 2, where we show the differences between the two values as a function of radial frequency. The fits for other values of the azimuthal frequencies or for other images of different galaxies are of similar quality and do not depend on the passband used.

Since we assume that each of these Gaussians corresponds to a different signal and that all signals add up to constitute a particular spectrum, we can recover their contribution to the galaxy image by simply Fourier transforming back each of the signals separately. The spectrum of a component must be a complex function as is the case for the spectrum of any $m$ component of the galaxy image, i.e.

$A(p, m)=|A(p, m)| \mathrm{e}^{i \phi(p, m)}$.

For each of the fitted Gaussian components, given a fixed value of $m$, we keep the same complex phase as in the transformed spectrum. Then the fitted spectra in the complex domain, can be expressed by

$A(p, m)=\sum_{j=1}^{N_{\mathrm{g}}} C_{j} \exp \left(\frac{\left(p-p_{j}\right)^{2}}{2 \sigma_{j}^{2}}\right) \mathrm{e}^{i \phi(p, m)}$.

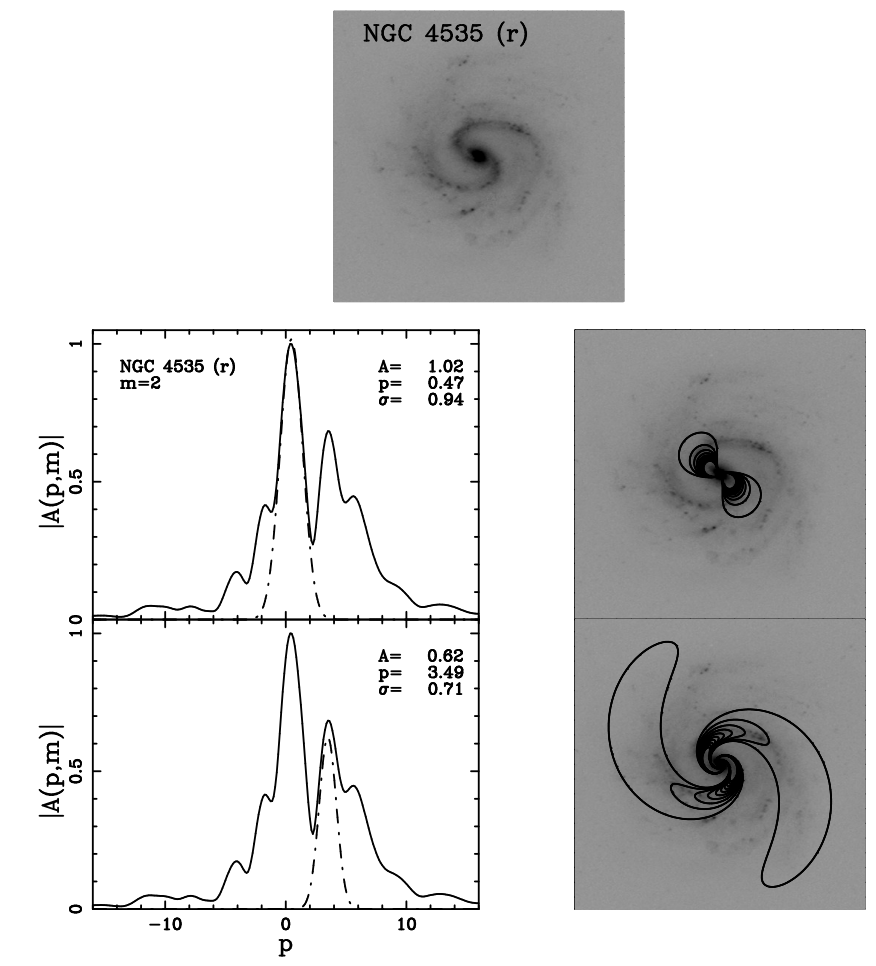

Fig. 3. Principal $m=2$ components for NGC 4535. The upper panel shows the deprojected galaxy viewed in the $r$ band. The second and third rows of panels concern the bar and the main spiral components, respectively. In the left we give the modules of the selected components (dot-dashed lines) and the total $m=2$ modulus (solid line). In the righthand panels we superpose the density of the component on the galaxy image. It is clear that these two components alone give a reasonable but not perfect reproduction of the main features of the galaxy. For a more detailed comparison, more components should be used.

Thus, the density of each component can be recovered through the following inverse Fourier transform:

$D_{j m}(u, \theta)=\frac{1}{4 \pi^{2} \mathrm{e}^{2 u}} \int_{-\infty}^{\infty} C_{j} \exp \left(\frac{-\left(p-p_{j}\right)^{2}}{2 \sigma_{j}^{2}}\right) \mathrm{e}^{\mathrm{i} \phi(p, m)} \mathrm{e}^{-\mathrm{i}(p u+m \theta)} \mathrm{d} p$,

where $D_{j m}(u, \theta)$ is the density distribution corresponding to the $j$ th Gaussian component of the azimuthal frequency $m$. In the second and third rows of panels in Fig. 3 we show the result of the inverse transformation of the two highest components in the $m=2$ spectrum of the $r$ band image of the galaxy NGC 4535 . The major peak corresponds to the bar component, while the second highest peak gives a two-armed structure. It can be noted, from this figure, that this component gives a general outline of the main two-armed spiral, but this is far from perfect. This is because the two-armed spiral seen in this particular image is the result of the superposition of all the components in this spectrum. This shows that to recover the details of a galaxy image, it is necessary to use a decomposition of the spectra including all the main signals. This procedure can be used to recover and analyse all the details of the spiral structure of a galaxy image. In this paper we concentrate exclusively in the bar signals and we leave the detailed analysis of the spiral structure for a forthcoming paper. 


\section{Samples and bar strength measures}

\subsection{The samples}

In this paper we work with two galaxy samples: the Frei sample (Frei et al. 1996) and the Ohio State University Bright Spiral Galaxy Survey (OSU; Eskridge et al. 2002). From these samples we only kept galaxies that are less inclined than $65^{\circ}$, do not have very luminous foreground stars projected on the image, and without strong irregularities. Thus we get 25 spiral galaxies in the $g, r$, and $i$ passbands and 35 galaxies observed in the $B_{J}$ and $R$ passbands from the Frei sample. For their deprojection we use the values found in Barberà et al. (2004).

The OSU provides deep, photometrically non-calibrated images of a complete magnitude limited sample of nearly 200 bright, nearby, well-resolved spirals. This survey selects galaxies with Hubble type S0/a or later with a magnitude in the blue $B<12$ and a diameter $D<6$ arcmin. For a number of these all colours $B V R J H K$ are available, while for a few some bands are still missing. From the OSU Early Data Release, we have taken only those galaxies with galaxy type $2 \leq t \leq 7$, which are less inclined than $65^{\circ}$ and for which we have position and inclination angles calculated with the same method as for the Frei sample (García-Gómez \& Athanassoula 1991). This gives a total of 128 galaxies for which we analyse the $B$ and $H$ images. All of these galaxies have already been classified as SA, SAB, and SB in the RC3 catalogue. Thus, in this paper, we use this standard classification for the barred galaxies of our sample.

We analysed all the images following the procedure outlined in Sect. 2. We also obtained the relevant spectra and decomposed the modulus of each single spectrum in its Gaussian components. Then, we were able to obtain all the spiral components in each of the $m$ Fourier spectra for all the galaxy images. This gives a wealth of information on the spiral structure for each galaxy. In Table 1 we show all the components in the $m=1,2$, and 3 spectra that are within the $25 \%$ of the maximum signal for all the example galaxies in this paper. We computed all the components in the following $m$ spectra: $m=1,2,3,4,6,8$ for all the galaxies; we plan to use this information to analyse the spiral structure of disc galaxies in a forthcoming paper. In Table 1, Col. 1 gives the NGC galaxy name, Col. 2 the filter used in the image, Col. 3 the corresponding $m$ Fourier spectra, and in Cols. 4-6, we give the parameters of the Gaussian components, namely their centre, peaks, and dispersion. The bar components are showed in boldface, as their discussion is the main objective of this paper.

\subsection{Measures of the bar strength}

We understand intuitively that stronger bars have more prominent bar components (i.e. bar Gaussians in the spectra). We have several ways of quantifying this fact using the measurements obtained with the FFT transform technique. We can choose to use the amplitude of the fitted Gaussian, its integrated modulus, or its power to characterise the bar strength. We use uncalibrated galaxy images and some caution must be taken when comparing the signals from different galaxies.

We examine the $m=2$ spectrum of each galaxy image to see whether there is a bar component or not. Such a component would necessarily have a peak within the range $-1.0 \leq p \leq 1.0$. The bar component, however, does not need to be the strongest signal in the $m=2$ spectrum. So, we define the bar component as the highest signal within this particular range. We designate its amplitude or central peak by $C_{\mathrm{B}}$ and its radial frequency by $p_{\mathrm{B}}$ while its dispersion is defined by $\sigma_{\mathrm{B}}$.
For each bar signal we then define the value of the bar modulus, hereafter $B_{M}$,

$B_{M}=C_{\mathrm{B}} \int_{-p_{\max }}^{p_{\max }} \exp \left(-\frac{\left(p-p_{\mathrm{B}}\right)^{2}}{2 \sigma_{\mathrm{B}}^{2}}\right) \mathrm{d} p$,

and similarly, we define the bar power as the value

$B_{P}=C_{\mathrm{B}}^{2} \int_{-p_{\max }}^{p_{\max }} \exp \left(-\frac{\left(p-p_{\mathrm{B}}\right)^{2}}{\sigma_{\mathrm{B}}^{2}}\right)^{2} \mathrm{~d} p$.

Either of these two values, $B_{M}$ or $B_{P}$, can be used to characterise the bar signal.

To avoid problems with non-calibrated images. All images are normalised to their maximum brightness, this is, the intensity value of the brightest pixel after cleaning up the images from all foreground stars and bad pixels. For all the galaxies in our sample this centring process is critical. If the images were not centred in this brightest pixel, we obtained a spurious contribution to the $m=1$ component clearly visible in the spectrum.

In this way, the $B_{M}$ and $B_{P}$ values can be used to compare bars in different galaxies. With this normalisation, these values are a relative measure of the importance of the bar signal within each galaxy. These parameters can also be further normalised to other galaxy properties, as are the power or the modulus in the $m=0$ or $m=2$ spectrum, or we can also use the sum of all the spectra combined. The $m=0$ component corresponds to the underlying galaxy disc. The normalisation provide measures of the bar that can be helpful to understand the bar strength in relation to other galaxy components and can also be used to relate the bar signals from different galaxies. We have to be careful with these normalisations, however, as a poorly defined disc in a galaxy image can considerably bias the value of some parameters for a particular galaxy when using their $m=0$ component in the normalisations.

Thus, the bar modulus can be normalised to the total modulus of the axisymmetric component $\left(m=0, B_{M 0}\right)$, the total modulus in the bi-symmetric component $\left(m=2, B_{M 2}\right)$, or to the total modulus of all the components combined $\left(B_{M \Sigma}\right)$, to obtain the bar parameters defined as in the following expressions:

$$
\begin{aligned}
& B_{M 0}= \frac{C_{\mathrm{B}} \int_{-p_{\max }}^{p_{\max }} \exp \left(-\frac{\left(p-p_{\mathrm{B}}\right)^{2}}{2 \sigma_{\mathrm{B}}^{2}}\right) \mathrm{d} p}{\int_{-p_{\max }}^{p_{\max }}|A(p, 0)| \mathrm{d} p} \\
& B_{M 2}=\frac{C_{\mathrm{B}} \int_{-p_{\max }}^{p_{\max }} \exp \left(-\frac{\left(p-p_{\mathrm{B}}\right)^{2}}{2 \sigma_{\mathrm{B}}^{2}}\right) \mathrm{d} p}{\int_{-p_{\max }}^{p_{\max }}|A(p, 2)| \mathrm{d} p} \\
& B_{M \Sigma}=\frac{C_{\mathrm{B}} \int_{-p_{\max }}^{p_{\max }} \exp \left(-\frac{\left(p-p_{\mathrm{B}}\right)^{2}}{2 \sigma_{\mathrm{B}}^{2}}\right) \mathrm{d} p}{\sum \int_{-p_{\max }}^{p_{\max }}|A(p, m)| \mathrm{d} p}
\end{aligned}
$$

and similarly the expressions:

$$
\begin{aligned}
& B_{P 0}=\frac{C_{\mathrm{B}} \int_{-p_{\text {max }}}^{p_{\text {max }}} \exp \left(-\frac{\left(p-p_{\mathrm{B}}\right)^{2}}{2 \sigma_{\mathrm{B}}^{2}}\right) \mathrm{d} p}{\int_{-p_{\text {max }}}^{p_{\max }}|A(p, 0)|^{2} \mathrm{~d} p} \\
& B_{P 2}=\frac{C_{\mathrm{B}} \int_{-p_{\text {max }}}^{p_{\max }} \exp \left(-\frac{\left(p-p_{\mathrm{B}}\right)^{2}}{2 \sigma_{\mathrm{B}}^{2}}\right) \mathrm{d} p}{\int_{-p_{\text {max }}}^{p_{\max }}|A(p, 2)|^{2} \mathrm{~d} p} \\
& B_{P \sum}=\frac{C_{\mathrm{B}} \int_{-p_{\max }}^{p_{\max }} \exp \left(-\frac{\left(p-p_{\mathrm{B}}\right)^{2}}{2 \sigma_{\mathrm{B}}^{2}}\right) \mathrm{d} p}{\sum \int_{-p_{\max }}^{p_{\max }}|A(p, m)|^{2} \mathrm{~d} p}
\end{aligned}
$$


Table 1. Parameters of the Gaussian components of the Fourier decomposition for the galaxies used in the text.

\begin{tabular}{|c|c|c|c|c|c|c|c|c|c|c|c|}
\hline NGC & Filter & $m$ & Centre & Peak & Disp & NGC & Filter & $m$ & Centre & Peak & Disp \\
\hline \multirow[t]{3}{*}{0210} & $B$ & 1 & 1.570 & 0.375 & 1.184 & 4303 & $r$ & 1 & -0.376 & 0.968 & 1.595 \\
\hline & & 2 & 0.661 & 0.992 & 1.278 & & & & 2.052 & 0.437 & 0.936 \\
\hline & & & -2.287 & 0.384 & 1.022 & & & & 4.042 & 0.325 & 0.669 \\
\hline \multirow[t]{3}{*}{0210} & $H$ & 1 & 0.172 & 0.495 & 1.334 & & & & -3.622 & 0.253 & 0.822 \\
\hline & & 2 & 0.426 & 0.980 & 1.394 & & & 2 & -0.068 & 0.641 & 0.960 \\
\hline & & & -2.140 & 0.355 & 1.063 & & & & -3.477 & 0.292 & 0.705 \\
\hline \multirow[t]{3}{*}{1073} & $B$ & 1 & 1.030 & 0.367 & 1.087 & 4535 & $g$ & 1 & -0.538 & 0.449 & 1.036 \\
\hline & & & -2.378 & 0.264 & 1.481 & & & 2 & 0.488 & 1.010 & 0.969 \\
\hline & & 2 & 0.005 & 1.004 & 1.227 & & & & 3.312 & 0.627 & 0.719 \\
\hline 1073 & $H$ & 2 & 0.010 & 1.013 & 1.325 & & & & -2.309 & 0.456 & 0.681 \\
\hline \multirow[t]{8}{*}{4303} & $B$ & 1 & -1.316 & 0.925 & 1.102 & & & & -4.125 & 0.305 & 0.789 \\
\hline & & & 1.368 & 0.701 & 0.694 & & & & 5.811 & 0.289 & 0.541 \\
\hline & & & 0.112 & 0.400 & 0.629 & 4535 & $i$ & 1 & -0.329 & 0.967 & 1.120 \\
\hline & & & -3.603 & 0.377 & 0.623 & & & & 1.789 & 0.385 & 0.922 \\
\hline & & & 3.184 & 0.302 & 0.577 & & & & -2.385 & 0.261 & 0.742 \\
\hline & & 2 & 0.802 & 0.521 & 0.733 & & & 2 & 0.468 & 0.998 & 0.914 \\
\hline & & & -3.607 & 0.385 & 0.684 & & & & 3.356 & 0.423 & 0.686 \\
\hline & & & -1.501 & 0.341 & 0.416 & & & & -2.190 & 0.287 & 0.696 \\
\hline \multirow[t]{3}{*}{4303} & $H$ & 1 & -0.045 & 0.981 & 1.491 & 4535 & $r$ & 1 & -0.270 & 0.498 & 1.899 \\
\hline & & 2 & 0.692 & 0.613 & 0.906 & & & & 0.997 & 0.473 & 1.204 \\
\hline & & & -2.120 & 0.248 & 0.487 & & & 2 & 0.626 & 0.899 & 0.928 \\
\hline \multirow[t]{11}{*}{4303} & $g$ & 1 & 0.161 & 0.972 & 0.926 & & & & 3.417 & 0.511 & 0.664 \\
\hline & & & 2.416 & 0.612 & 0.831 & & & & -2.076 & 0.345 & 0.563 \\
\hline & & & -2.064 & 0.451 & 0.776 & & & & 5.880 & 0.225 & 0.594 \\
\hline & & & 4.417 & 0.310 & 0.581 & 5247 & $B$ & 1 & 0.256 & 1.012 & 1.289 \\
\hline & & 2 & 0.766 & 0.792 & 1.033 & & & & -2.746 & 0.489 & 0.848 \\
\hline & & & -3.553 & 0.774 & 0.753 & & & & 5.443 & 0.270 & 1.168 \\
\hline & & & -1.463 & 0.428 & 0.413 & & & 2 & -2.991 & 0.949 & 0.863 \\
\hline & & & -5.695 & 0.292 & 0.758 & & & & -1.061 & 0.761 & 0.629 \\
\hline & & 3 & -1.533 & 0.276 & 0.736 & & & & 0.999 & 0.384 & 0.799 \\
\hline & & & -3.418 & 0.254 & 0.516 & & & & 2.599 & 0.292 & 0.786 \\
\hline & & & -5.479 & 0.245 & 0.568 & & & 3 & -2.275 & 0.547 & 1.045 \\
\hline \multirow[t]{6}{*}{4303} & $i$ & 1 & -1.211 & 0.588 & 1.125 & 5247 & $H$ & 1 & -0.392 & 0.991 & 1.157 \\
\hline & & & 0.460 & 0.375 & 1.212 & & & 2 & -0.875 & 0.497 & 0.640 \\
\hline & & & 1.477 & 0.374 & 0.771 & & & & -3.454 & 0.469 & 0.708 \\
\hline & & & -3.719 & 0.321 & 0.805 & & & & 2.509 & 0.262 & 1.231 \\
\hline & & & 3.536 & 0.306 & 0.708 & & & & & & \\
\hline & & 2 & 0.425 & 0.986 & 1.188 & & & & & & \\
\hline
\end{tabular}

for the case of the power of the components. We note that $\sum$ means that $m=0,1,2,3,4,6,8$. We normally exclude the $m=5$ component, which is usually a flat component, from our analysis.

\section{Application to model bars and to a few fiducial barred galaxies}

\subsection{Application to model bars}

In order to better understand our results, we first applied our method to model galaxies, composed simply by adding an exponential disc and a Ferrer two-dimensional bar. The density distribution of the latter is given by the expression

$\rho(x, y)=\left\{\begin{array}{rr}(1-d(x, y))^{n}: & d(x, y)<1 \\ 0: & d(x, y) \geq 1\end{array}\right.$

where

$d(x, y)=\left(\frac{x}{a}\right)^{2}+\left(\frac{y}{b}\right)^{2}$

$a$ and $b$ are the major and minor axes of the bar and $n$ is the concentration index describing how fast the density decreases with 
C. Garcia-Gómez et al.: Measuring bar strength using Fourier analysis of galaxy images

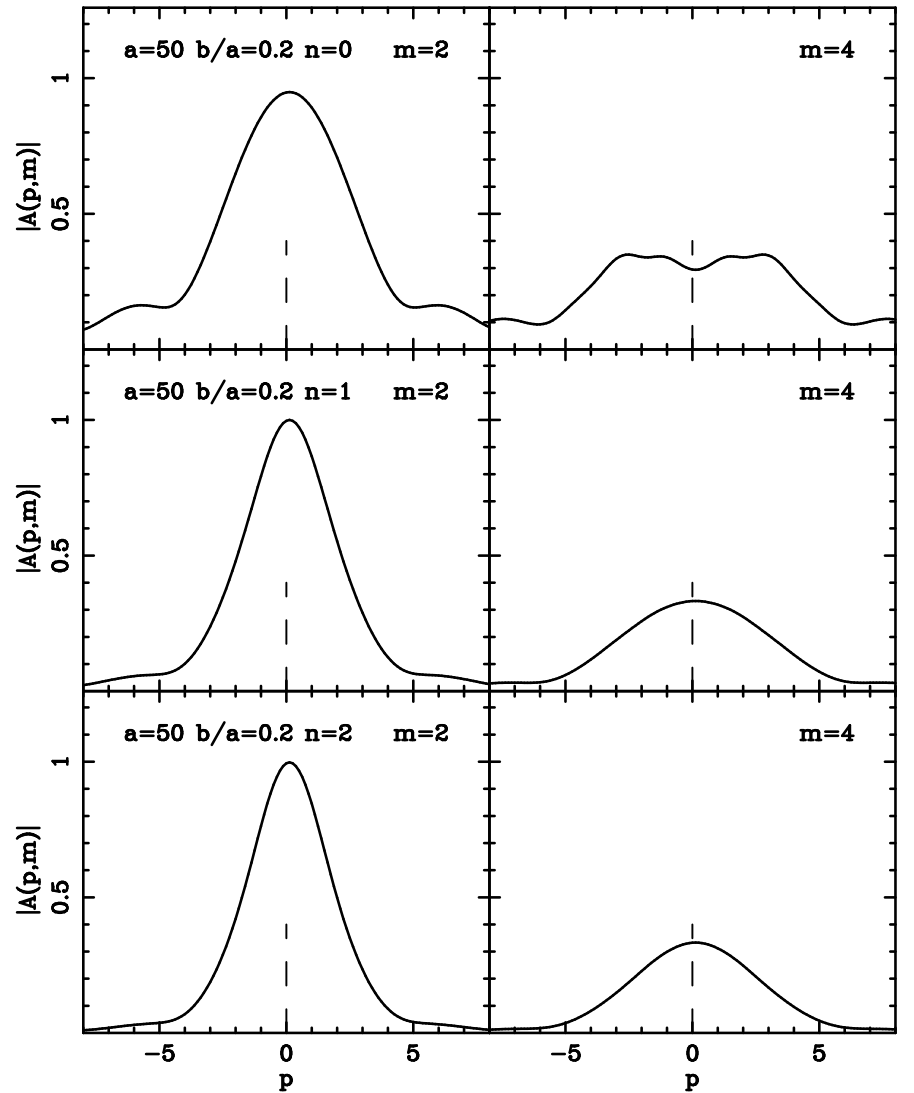

Fig. 4. Spectra from the idealised barred galaxy images with different concentration indexes and an axis ratio of 0.2. The results for the $m=$ 2 components are given in the left-hand panels and for the $m=4$ in the right-hand panels. A dashed vertical line is placed at $p=0$. The numerical values of $a, b$, and $n$ are given in the upper left corner of the left panels. The three signals are normalised to the maximum of the three values.

increasing distance from the centre. We always use an exponential disc with a scale length of 20 units, while we change at will the values of the axes ratio $a / b$ and the concentration index $n$.

Figures 4 and 5 show the $m=2$ and $m=4$ spectra of different idealised bars embedded within an exponential disc. In Fig. 4 we show the effect of different values of the concentration index $n$ on the shape of the bar signal. The signals are normalised to the maximum fiducial value of the three units to facilitate the comparison. We can see that higher values of $n$ give bar signals having narrower profiles, but similar strengths. The secondary maxima that appear at both sides of the bar signal are entirely artificial because the combined intensity of the artificial bar and disc has a jump at the ends of the bar. In all cases, however, the modulus of the main components in the $m=2$ spectrum has a bell shape and is centred at $p=0$. The effect of increasing the bar axial ratio while keeping the bar concentration index constant is shown in Fig. 5. We can see in this figure that round bars give rounder signals, i.e. with larger dispersion and more extended wings. Also, as the bar becomes rounder, the relative importance of the signals is lower. This kind of signal can be easily lost when combined with strong arms. Thus, the clean separation of signals is an important step in the study of the spiral structure of galaxies. Strong bars, on the other hand, are better represented by model bars with a concentration index that is larger or equal to $n=2$ and axis ratios with values $(b / a)<0.5$.

Of course, these cases are very idealised. Owing to various irregularities or asymmetries, galactic bars do not necessarily

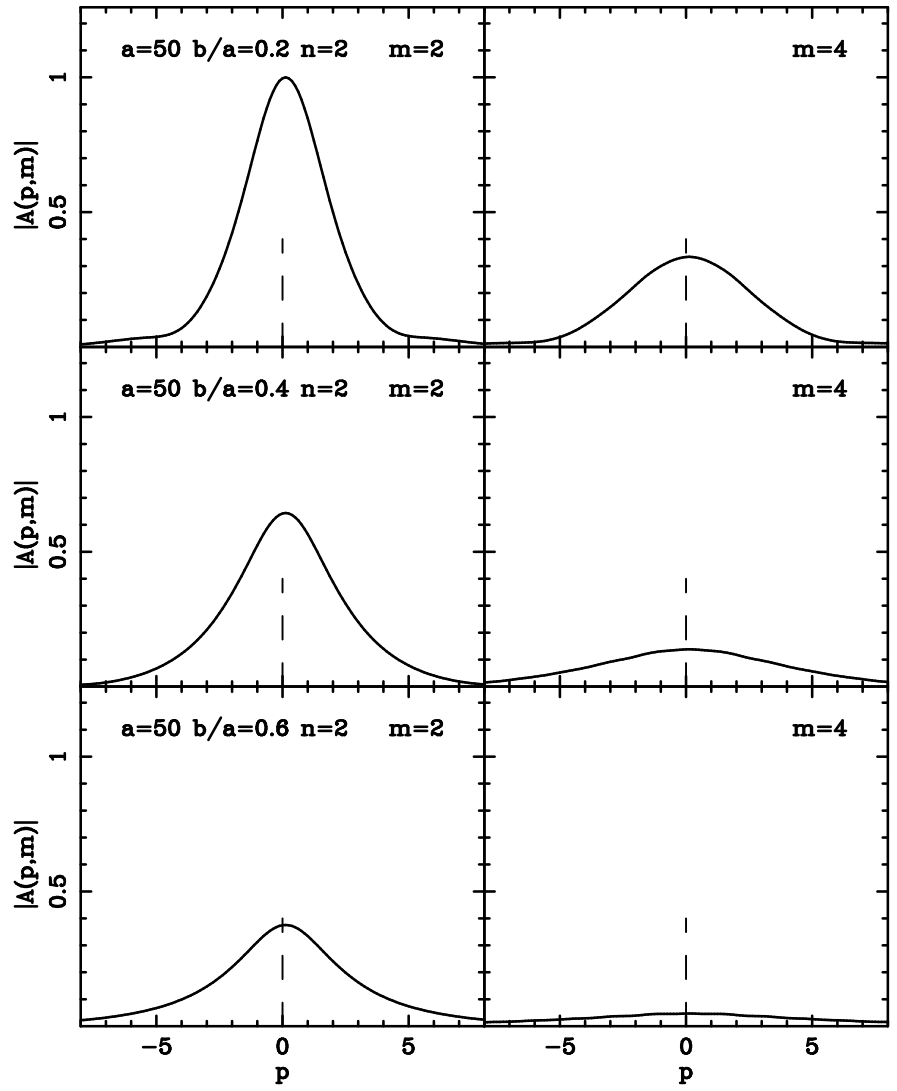

Fig. 5. Spectra from the idealised barred galaxy images with different bar axis ratio and an $n=2$ concentration index. The results for the $m=2$ components are given in the left-hand panels and the $m=4$ in the right-hand panels. A dashed vertical line is placed at $p=0$. The three signals are normalised to the maximum of the three values.

give signals that are symmetric with respect to their maximum. The modulus of the spectrum then requires more components to be properly described. This was the case for NGC 4535, in Fig. 3 , where the main spiral was not entirely logarithmic and more than the single maximum component was necessary to give a proper description of the $m=2$ spectrum. Moreover, spirals often start off from the end of the bar, so the location of the maximum may be somewhat offset from $p=0$. The Gaussian corresponding to the bar component is hereafter called the bar Gaussian, for brevity.

\subsection{Application to a few fiducial barred galaxies}

In this paper we focus on the detection and classification of bar structures in galaxy discs. The standard galaxy classification of barred galaxies used by de Vaucouleurs (1963) distinguishes three families of disc galaxies: SA, SB, and SAB. Galaxies in the SA family have no bars, SB galaxies have a clear bar component, while $S A B$ galaxies are intermediate. In practice, this means that the SAB family is a mixed bag. It contains galaxies with fat ovals, galaxies with short bars, or simply unclear cases. In the more recent RC3 catalogue by de Vaucouleurs et al. (1991), this galaxy classification is maintained and it will be followed in this paper. In this section we apply our technique to four disc galaxies with different morphologies for the bar structures that have been classified accordingly in the RC3 catalogue. This allow us to check whether our method based on the analysis of the Fourier spectra can be used to identify bars and measure bar strengths in 


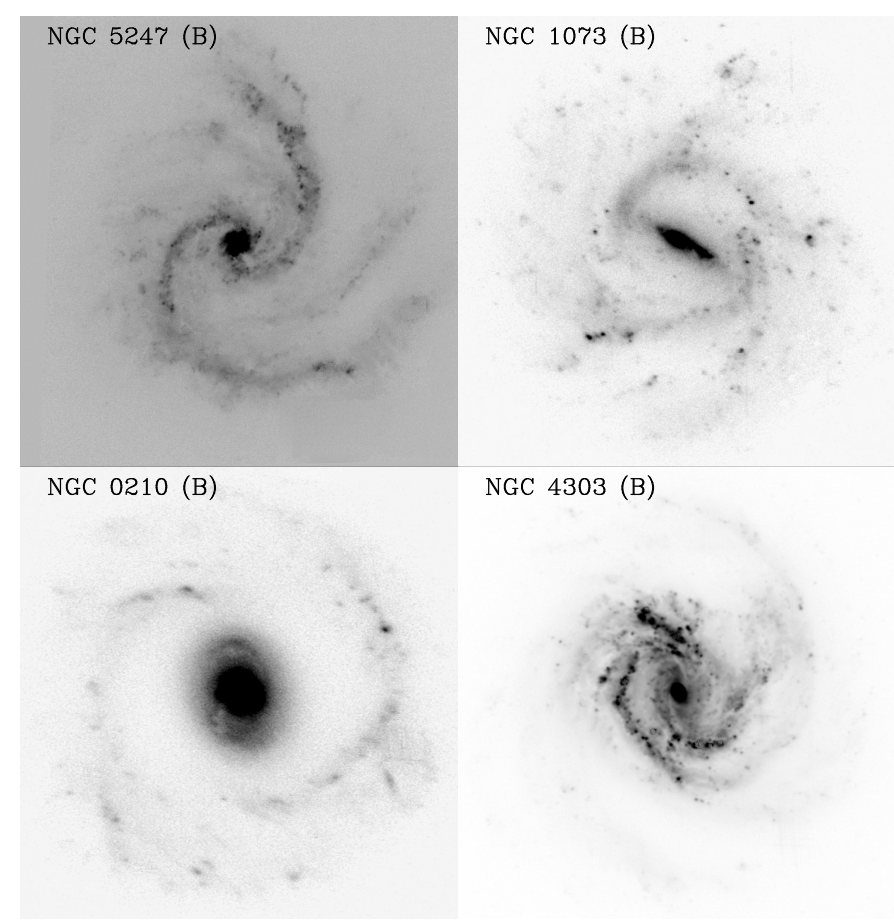

Fig. 6. Blue images of the four analysed galaxies: NGC 5247 (upper left), NGC 1073 (upper right), NGC 210 (lower left) and NGC 4303 (lower right).

disc galaxies. We show images in the blue passband of these four galaxies in Fig. 6.

A visual examination of NGC 5427 does not reveal any bar and, thus, this galaxy has been classified as SA in the RC3 catalogue. On the contrary, NGC 1073 is classified as SB in the RC3 catalogue and shows a clear strong bar structure. We have also included the galaxies NGC 210 and NGC 4303, both of which are classified as SAB in the RC3 catalogue, showing different types of bar or oval structures. Indeed, NGC 210 has a clear oval structure, while NGC 4303 has a short bar. The features in the spectra of the $B$ passband are also clearly visible in the spectra from the $H$ filter for the same galaxies. In Fig. 7 we show the galaxy images in the $H$ passband. Although the spiral arms are now dimmer and smoother than in the $B$ band, the main galaxy structures are still clearly visible.

In Fig. 8 we show, in the first column, the $m=2$ spectra for these four galaxies as calculated from the $B$ passband images while in the second column we show the same spectra obtained using $H$ passband images. As we can see, the spectra of all these galaxies are very complex. Only the $m=2$ spectrum of the strongly barred SB galaxy NGC 1073 shows a strong signal centred at $p=0$. Even in this case, the spectrum is not perfectly symmetric, however. In the case of NGC 5247, we have a very broad $m=2$ spectrum, which is the result of the combination of the central structure and the asymmetric multiple strong arms, which give signals for different values of the radial frequency $p$. In the case of the strongly barred galaxy NGC 1073 the spectrum shows indeed a strong signal, approximately centred on $p=0$. This galaxy also shows an asymmetrical two-armed spiral, which appears in the spectrum as a very small peak on the right side of the main peak, as well as some other minor components. All of these contribute to the $m=2$ spectrum and their blending finally gives a strong signal that is not perfectly centred on $p=0$. This blending is clearly seen in the $m=2$ spectrum

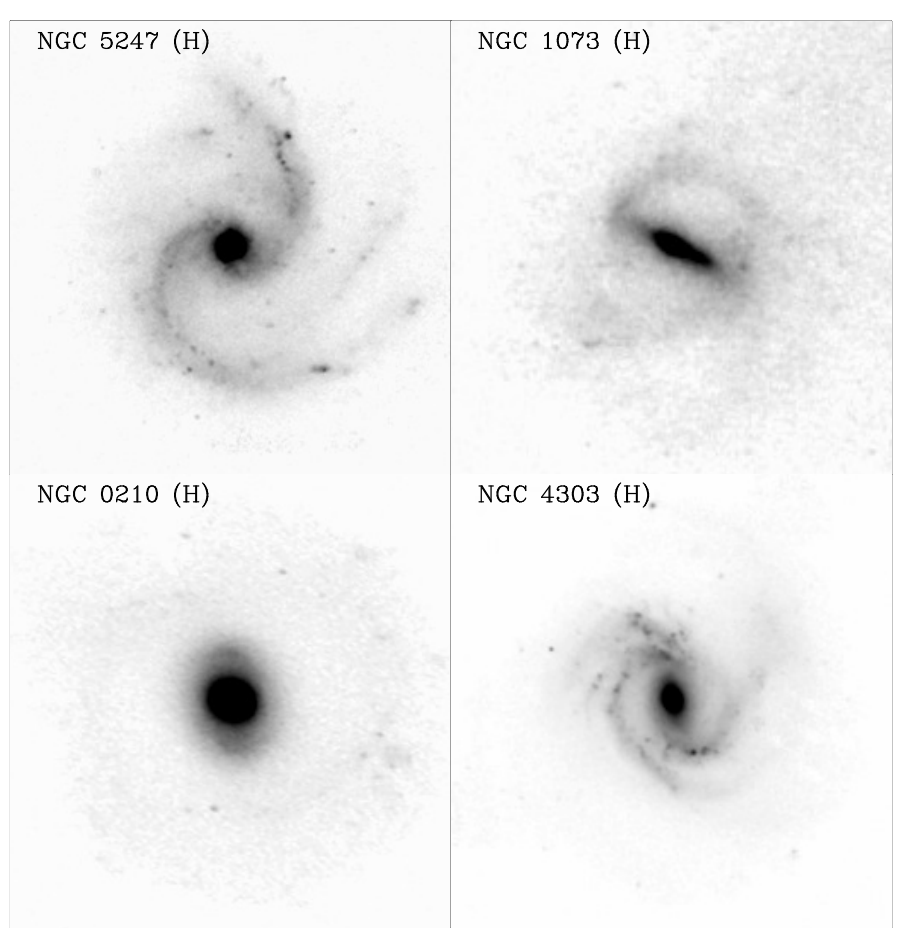

Fig. 7. Near-infrared images of the four galaxies: NGC 5247 (upper left), NGC 1073 (upper right), NGC 210 (lower left) and NGC 4303 (lower right).

of NGC 210, whose image shows a strong oval and rather dim spiral arms. The $m=2$ spectrum of this galaxy shows an asymmetric strong peak that is not centred at $p=0$, resulting from the blending of the oval signal with the signal from the dim spiral arms. NGC 4303, whose image shows stronger arms and a more complex structure, also has a broad spectrum that is the combination of all these components. As in the case of the $B$ passband, the $H$ image of NGC 5247 shows the broken arms, which give two strong signals with no apparent bar. For the case of NGC 1073 the strong bar signal is biased by the dim bar signal of the arms giving an asymmetric composed signal. Finally, the combined signals of the bar structure and the arms give also complex signals in the $m=2$ spectra for the galaxies NGC 210 and NGC 4303. Comparing these two sets of spectra we can see that while the shape of the spectra can be somewhat different using two different passbands, when decomposing the spectra and obtaining all the signals separately, there are no great differences in the relevant components detected in all the passbands. We can conclude that in general the spiral or bar features of a galaxy are common to $B$ and $H$ passband, as is the case for these examples. This result suggests that the passband used by the Fourier technique for the detection of bar and spiral components is not a decisive factor and that the bar or spiral components can be normally detected in all the passbands.

Finally, we can study the performance of our method, when using images with lower resolution coming from deep samples of cosmological interest. For this analysis we select the strongly barred galaxy NGC 1073 because in this particular case our method gives a well-centred strong signal in the $m=2$ spectrum corresponding to the bar, while the dimmer two-spiral structure is also represented by a smaller signal. We start with the NGC 1073 galaxy image from the OSU galaxy sample using the $B$ passband. We degraded the resolution of the image successively by a factor 2 and recalculated its $m=2$ spectrum for each image. Next we can study the effect of the reduction of the 


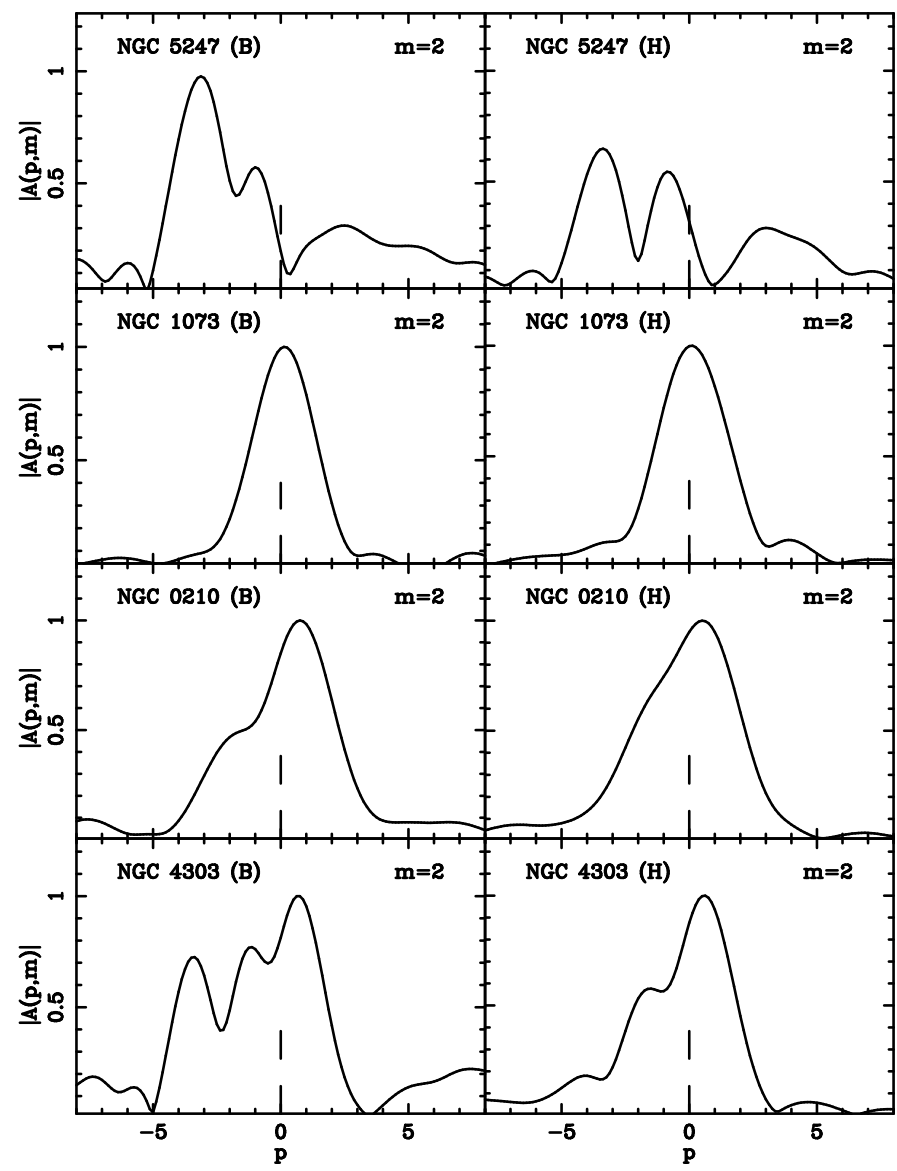

Fig. 8. Spectra from the $B$ and $H$ band images of the four galaxies shown in Figs. 6 and 7. From top to bottom, NGC 5247, NGC 1073, NGC 210, and NGC 4303 are shown. We can compare the results for the $m=2$ components in both passbands. In the left panels we have the spectra for the $B$ passband, while in the right panels the $m=2$ spectrum for each galaxy in the $H$ passband. A dashed vertical line shows the location of $p=0$.

image resolution on the signal of the bar and spiral component. The initial image has a resolution of $640 \times 640$ pixels, while we degrade down the resolution successively until we reached a final resolution of $20 \times 20$ pixels, i.e. a reduction in resolution by a factor 32. We show the result of this analysis in Fig. 9. The left column shows the successively degraded images of NGC 1073, starting from the initial image with $640 \times 640$ pixels. In the right column, we plot the signal of its corresponding $m=2$ spectrum. We show only three additional images with resolutions reduced by factors 4,16 , and 32 . Lowering the resolution is equivalent to placing this same galaxy at longer distances by the same factors. Comparing the original signal of the $m=2$ spectrum with the spectra of the lower resolution images, we can observe that our method is able to recover both the strong bar signal and the lower signal corresponding to the dimmer two arm structure, even at such lower resolutions. Thus, we can be confident that our method will be also useful with samples of cosmological interest with galaxies located much father away. This opens the possibility of studying the evolution in time of the spiral structure of galaxies via larger and deeper samples. The main problem with such studies, however, will be the requirement to use images with a well-defined disc, which is an essential step to obtaining a reliable deprojection of the image.

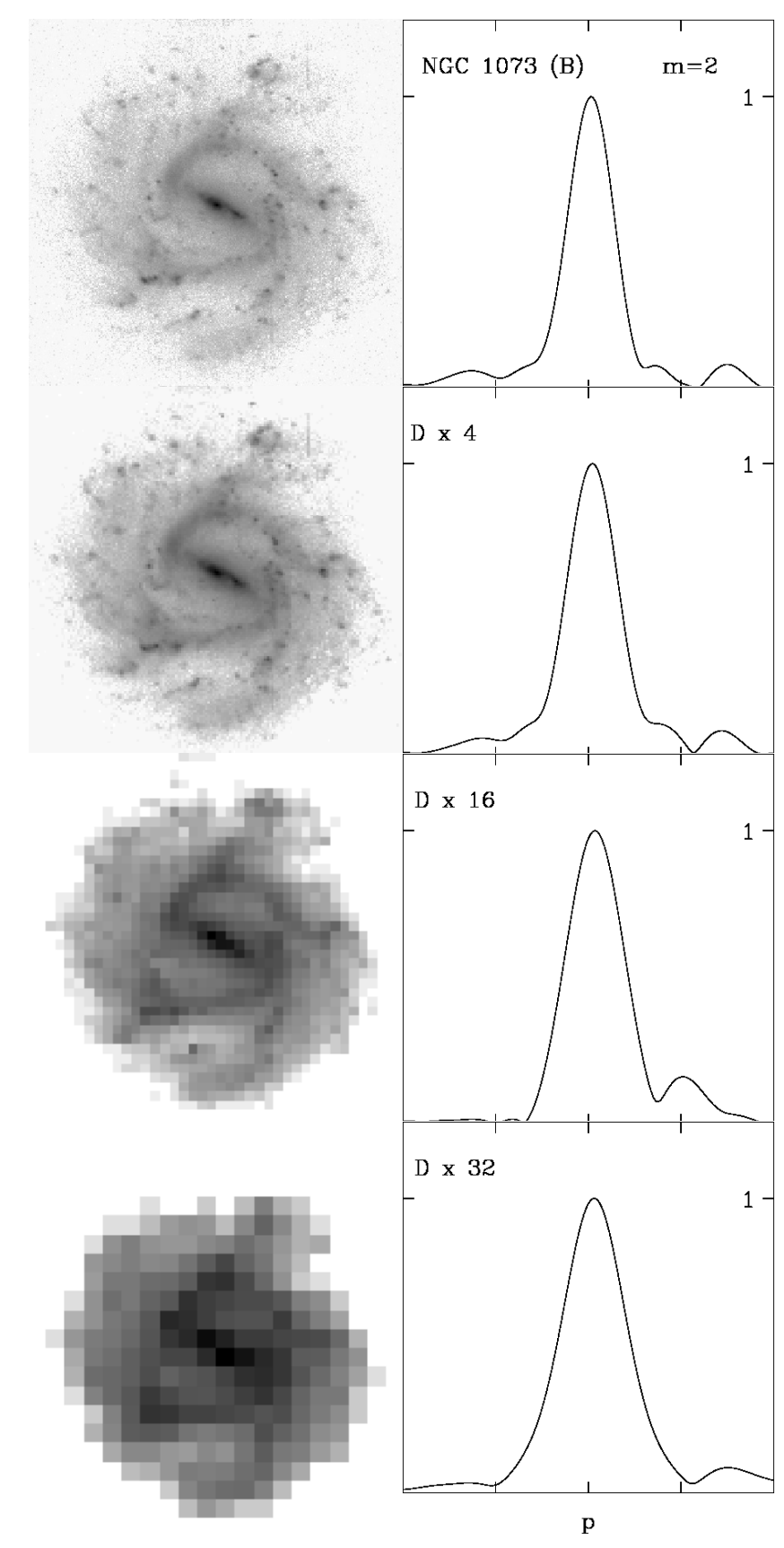

Fig. 9. Starting with the initial image in the $B$ passband for NGC 1073, in each row, results of the degraded resolution in the left column and the resulting $m=2$ spectrum of this degraded image by reducing resolution factors of 4,16 , and 32. We can recover the main features of the $m=2$ spectrum even at very low resolutions.

\section{Bar indicators as a function of passband}

For each galaxy, we have at least two images in different passbands. One of these is located in the blue region and the other in the red or NIR region. Thus, in the case of the Frei sample we use the $g$ or $B$ passbands for the blue and we use the $r$ or $R$ passbands for the red or NIR. In the case of the OSU sample, we use the $B$ filter for the blue region, while we use the $H$ filter for the red or NIR. For all the images for which we detected a signal, we can compare the strength in the two passbands. This procedure can show whether bars are in fact more prominent in the red or NIR than in the blue region as has been claimed by some authors. We used all of our bar indicators to compare the 

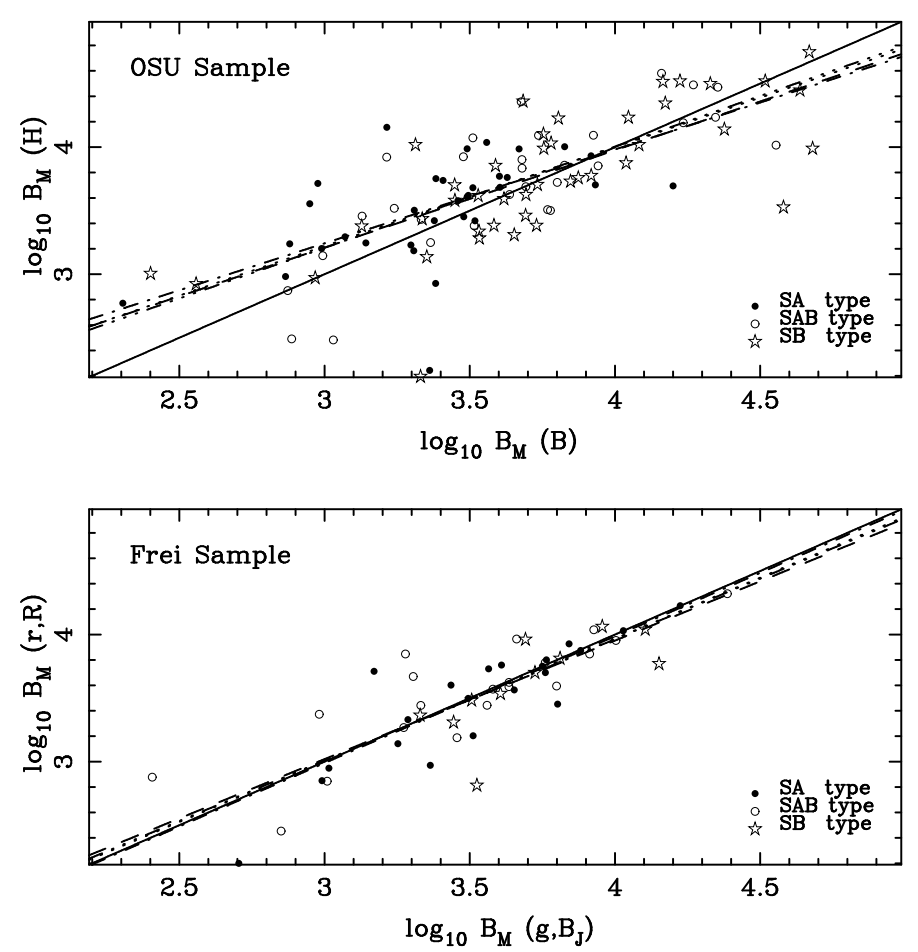

Fig. 10. Correlation between the values of the $\log _{10} B_{M}$ parameter for the blue and red filters. The bold line is the diagonal, while the dashed line is the standard least squares bisector line. The dot-dashed, dotted, and dot-dot-dot-dashed line correspond to the robust optimal Mregression line, the robust mean biponderate regression line, and the robust winsorised regression line, respectively. The $S A$ galaxies are represented as filled circles, the $S A B$ galaxies as open circles, and the $S B$ galaxies as open stars. The upper panel gives results for the OSU sample and the lower panel for the Frei sample.

strength of the bar signal in different passbands and all of these indicators give comparable results. We discuss here only some of these indicators for the sake of brevity.

In Fig. 10 we show the linear regression of the parameter $\log _{10} B_{M}$ for the blue and red passbands for both samples. For the linear regression we can compute the standard regression line and we can also perform robust regressions, which are less biased by the presence of outliers. Here we include the optimal M-regression line, the mean biponderate regression line and the winsorised regression line, as suggested by Huber (1981). The figure shows that all the linear correlations give similar results, and we can conclude that our samples are not strongly biased by outlier galaxies. We can also perform a significance test using a bootstrap technique on the values of the slope for the standard regression line and for the optimal M-regression line. In the case of the Ohio sample, the $95 \%$ confidence interval for the slope of the standard regression line is $[0.61,0.93]$, while the same interval for the optimal M-regression line is [0.63, 0.93]. Both intervals exclude the value $a=1$ for the slope of the regression line and we can conclude that bars in the $B$ passband give stronger values for our indicators than the same values in the $H$ passband for the Ohio sample galaxies. The same confidence intervals for the Frei sample give values of $[0.71,1.17]$ and $[0.77,1.19]$. Here, both intervals include the value $a=1$ for the slope and, in this case, there is no significant difference between the values of the bar indicators in the blue passband and red passband. Thus, our results suggest that bars in the far-infrared ( $H$ passband) appear dimmer with respect to the background galaxy than the same bars in the blue or red passbands. We obtained similar results using the $B_{P}$
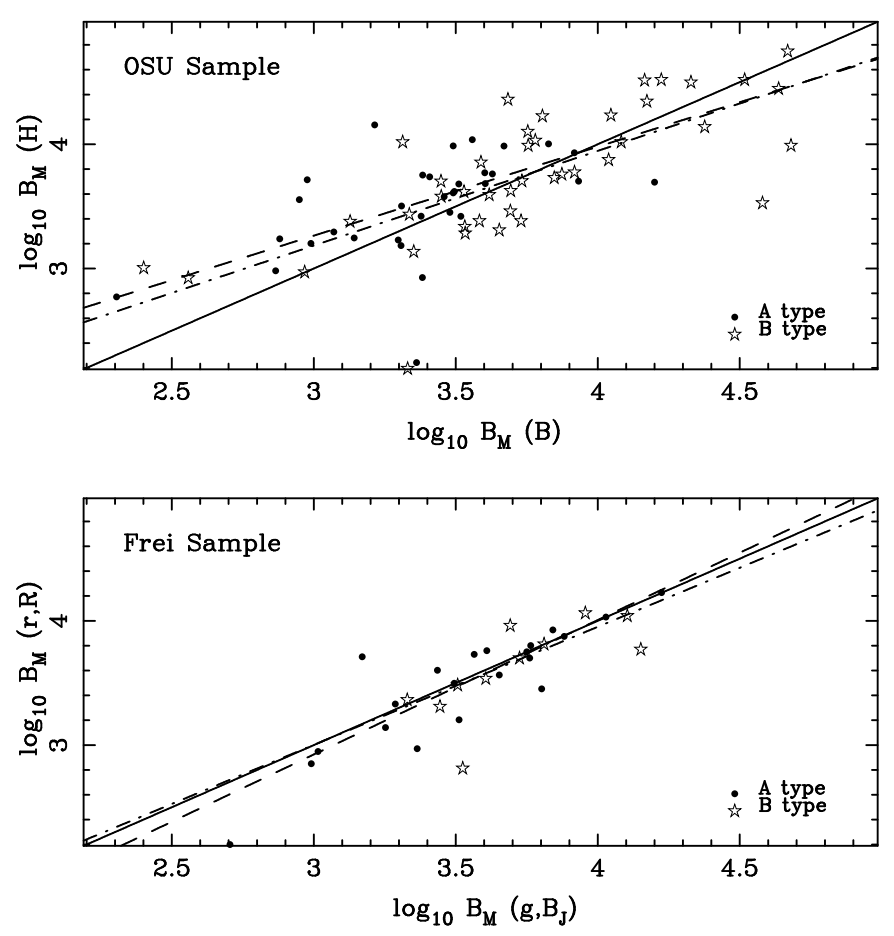

Fig. 11. Correlation between the values of the $\log _{10} B_{M}$ parameter for the blue and red passbands for the $S A$ and $S B$ galaxies separately. The layout is the same as in Fig. 10. The solid line corresponds to the diagonal, while we show only the optimal M-regression line for $S A$ (dashdotted line) and $S B$ (dashed) galaxies separately.

indicator instead of the $B_{M}$ indicators and, in this case, the $95 \%$ confidence intervals for the standard regression line in the Ohio sample is $[0.61,0.91]$ and the same interval for the optimal Mregression line is $[0.63,0.97]$. In the case of the Frei sample both intervals are $[0.74,1.16]$ and $[0.80,1.22]$, respectively.

The fact that the values of the slopes are very near unity in all the cases indicates, moreover, that for the galaxies with a signal detected in both passbands, there is no evidence of a significant difference between bar strength in different passbands. However, not all galaxy images in our sample showed a bar signal using our definition. All the galaxy images from the Frei sample showed bar signals in both passbands. For the galaxy images selected in the Ohio sample, however, only three galaxies from the 128 selected, namely NGC 4051, NGC 4580, and NGC 4699, failed to show a bar signal in any of the passbands at all, while 16 galaxies failed to show a bar signal at least in one of the two passbands. Five of them did not show a bar in the $H$ image and the rest of 11 galaxies only showed a bar signal in the $B$ band. We have to emphasise, however, that in these cases, we could have detected a bar signal if we had used a larger interval for the definition of the bar signal. In fact the interval selected, $(-1 \leq p \leq 1)$ (see Sect. 3.2), is somewhat arbitrary. If we relax this condition, we can detect a bar signal in both passbands.

Some authors (Laurikainen \& Salo 2002; Marinova \& Jogee 2007) have suggested that bars can be more easily detected in the infrared passbands than in the blue region of the spectrum. We used our bar indicators to compare the difference between $S A$ and $S B$ galaxies for the different passbands in our sample to check this result. The results are compared using the robust optimal M-regression line separately for the $S A$ and $S B$ galaxies in Fig. 11, where we compare the values of the $\log _{10} B_{M}$ parameter for the blue and red filters. There is no apparent difference between $S A$ and $S B$ galaxies, and a bootstrap analysis of the 

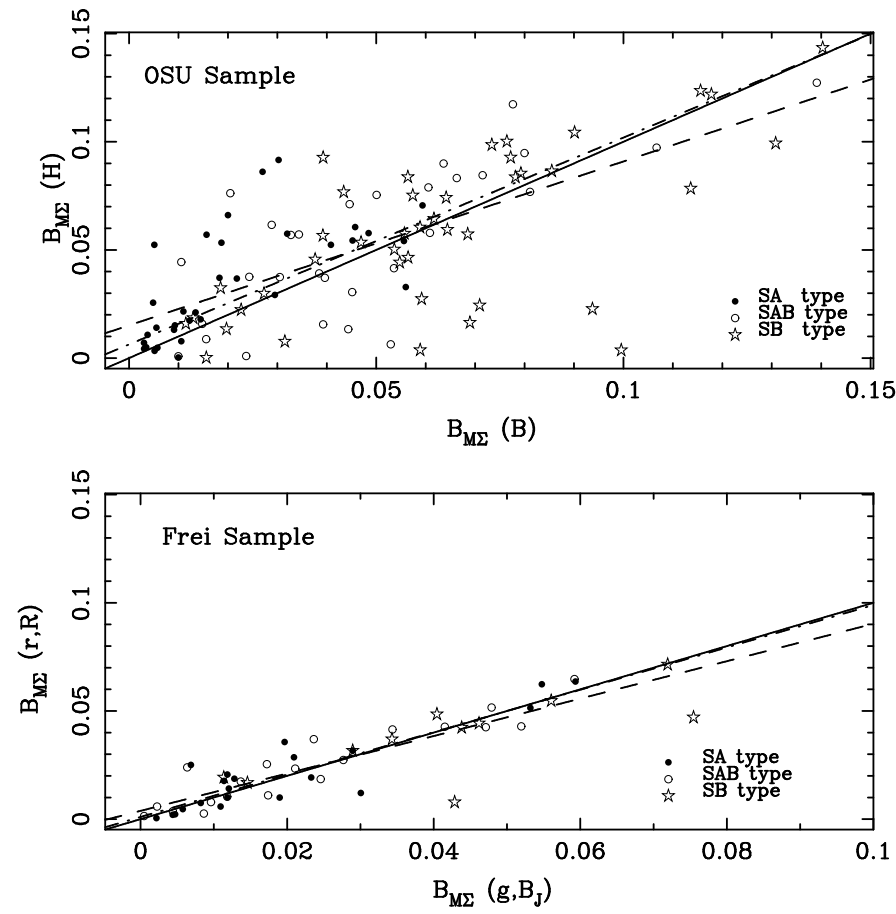

Fig. 12. Correlation of the values of the $B_{M \Sigma}$ parameter for the blue and red passbands for the two samples. The layout is as in Fig. 10, but here we only show the standard linear regression line (dash-dotted line) and the robust optimal M-regression line (dashed line).

slopes at a $95 \%$ confidence level confirms this result. The interval for $S A$ galaxies in the Ohio sample is [0.22, 0.97], while for $S B$ galaxies this interval is $[0.50,1.10]$. In the case of the Frei sample, the interval for $S A$ galaxies is $[0.91,1.42]$, while the interval for $S B$ galaxies is $[0.46,1.55]$. Thus, our data does not show any significant difference between $S A$ and $S B$ galaxies when using our bar indicators. We can conclude that when we use the Fourier technique there are no apparent differences in the bars detected for a particular galaxy using different passbands. These results are only valid for the set of passbands used in the galaxy samples selected for our analysis. It would be useful to extend the comparison of the bar strength to other passbands, such as the UV, using this technique to ascertain if there is some particular passband where the bars are more prominent.

In order to compare the bar signals we can also use the normalised values of our parameters described in Sect. 3.2. These normalised values, however, must be used with caution. For a particular galaxy with very well-defined arms or a poorly defined disc, the normalisation process can give a very biased indicator value. However, the results using the rest of the indicators are very similar to the results already shown in this section. In Fig.12 we show the correlations for one of the normalised parameters, namely the $B_{M} \sum$ parameter. Here we only show the standard linear regression line (dash-dotted line) and the robust optimal Mregression line (dashed line). The $95 \%$ confidence intervals for the slope do not show a significant difference between the bar indicators in the blue and red passbands. In the case of the Ohio sample these intervals are $[0.57,0.90]$ for the standard regression line and $[0.80,1.02]$ optimal M-regression line. In the case of the Frei sample these intervals are $[0.67,1.02]$ and $[0.85,1.00]$, respectively.

Using the normalised indicators, however, it is true that the values of the bar components for non-barred galaxies, as classified in the RC3 catalogue, are higher in the infrared than in the blue passbands. This is apparent in Fig.12. This would be in agreement with the claim that disc galaxies appear to be more barred in the infrared than in the visible passband. On the other hand, galaxies classified in the RC3 catalogue as barred, appear to have higher values of the indicators in the blue passband than in the red. The regression lines fail to give any significant difference between $S A$ and $S B$ galaxies. We will explore this result further in Sect. 7 using the distribution of the values of the normalised indicators.

\section{Comparison between different bar indicators}

We can find two ways of measuring the bar strength in the literature. The first is a measure of the maximum relative non-axisymmetric torque strengths (hereafter $Q_{\mathrm{b}}$ ) introduced by Combes \& Sanders (1981). This parameter was used by Block et al. (2002), Buta et al. (2004, 2005), and more recently by Speltincx et al. (2008). The second method, used by Whyte et al. (2002) and Marinova \& Jogee (2007), is to give a measure of the bar ellipticity. These authors have concentrated their efforts in the OSU sample.

The problem of detecting a bar component has been addressed by different authors who have suggested indicators based on particular properties of the bar component. In this section we want to compare the performance of these indicators on the galaxies in the OSU sample, which has been used for different groups to measure bar strengths. We compare the relative efficiency of our bar indicators, namely $B_{M}$ and $B_{P}$, with the $f_{\text {bar }}$ indicator used by Whyte et al. (2002), the bar torque $Q_{\mathrm{b}}$ used by Block et al. (2001), Buta et al. (2004), and Speltincx et al. (2008) and, finally, the ellipticity $\epsilon_{\mathrm{bar}}$ used by Marinova \& Jogee (2007).

As the bar indicators measure different bar properties, they are not directly comparable and a linear correlation does not seem to be an appropriate way to compare the results of the different groups of authors. Instead of the classical Pearson linear correlation coefficient, the Spearman and Kendall correlation coefficients seem to be more suitable for this study. These nonparametric coefficients are more appropriate for the case of samples that are drawn for unknown distributions; see Press et al. (1994).

Given a set of values $x_{i}$, we define the rank among these samples as $R_{i}$. If we have a second sample $y_{i}$ drawn from a different probability distribution, we define the rank within this sample as $S_{i}$, then the Spearman rank correlation coefficient $r_{\mathrm{s}}$ is defined by

$r_{\mathrm{s}}=\frac{\sum_{i}\left(R_{i}-\bar{R}\right)\left(S_{i}-\bar{S}\right)}{\sqrt{\sum_{i}\left(R_{i}-\bar{R}\right)^{2}} \sqrt{\sum_{i}\left(S_{i}-\bar{S}\right)^{2}}}$,

where $\bar{R}$ and $\bar{S}$ are the mean values of each sample. The values of this parameter must be within the range $-1 \leq r_{\mathrm{s}} \leq 1$, and the closer the value of $\left|r_{\mathrm{s}}\right|$ to 1 the better the correlation. The significance of these values against the null hypothesis of zero values can be tested by computing the parameter

$t=r_{\mathrm{s}} \sqrt{\frac{N-2}{1-r_{s}^{2}}}$,

which is distributed approximately as a $t$-Student distribution with $N-2$ degrees of freedom. This approximation does not depend on the unknown original distribution of the data values, 
which makes this method very appropriate to our purpose because the probability distribution of the values of the bar indicators is not known.

On the other hand, the Kendall $\tau$ coefficient uses only the relative ordering of ranks. Given $N$ data points $\left(x_{i}, y_{i}\right)$, we consider all the possible $N(N-1) / 2$ pairs of data points. A concordant pair occurs if the relative ordering of the $x_{i}$ values is the same as the relative ordering of the $y_{i}$ values, while a discordant pair occurs on the contrary. In the case of ties, the pair is not considered as concordant or discordant and the pair is taken as an "extra $y$ pair" if the tie is in the $x$ values or an "extra $x$ pair" if the tie is in the $y$ values. If $N_{\mathrm{c}}$ is the number of concordant pairs, $N_{\mathrm{d}}$ the number of discordant pairs, $E_{x}$ the number of extra $x$ pairs, and $E_{y}$ the number of extra $y$ pairs, the Kendall $\tau$ coefficient is defined as

$\tau=\frac{N_{\mathrm{c}}-N_{\mathrm{d}}}{\sqrt{N_{\mathrm{c}}+N_{\mathrm{d}}+E_{y}} \sqrt{N_{\mathrm{c}}+N_{\mathrm{d}}+E_{x}}}$.

This value lies in the range $[-1,1]$, taking extreme values only in the case of complete rank agreement or complete rank reversal, respectively. The approximate distribution or the $\tau$ coefficient in the null hypothesis of no correlation is approximately normally distributed with zero mean value and a variance of

$\operatorname{Var}(\tau)=\frac{4 N+10}{9 N(N-1)}$.

We use all these parameters with a significance level of $\alpha=0.95$ to compare all the measurements of bar strength. In the upper diagonal of Table 2, first we show the number of galaxies in common within the OSU sample, and then the values of the Pearson correlation coefficient, Spearman correlation test, and Kendall $\tau$ coefficient for each pair of authors. We also include the correlations between the values of our two indicators, $B_{M}$ (first row) and $B_{P}$, and the comparison between the indicators used for the rest of the research groups. We include in the comparison only two of our bar indicators as we obtain comparable results using the rest of our bar indicators. In the cases where both $B$ and $H$ images were used, we combine all the values to strengthen the correlation. In the lower diagonal, we show the respective $p$ values of the three correlation coefficients for this particular sample. The $p$ values lower than $\alpha=0.95$ indicate a significant correlation up to this level of significance. The $p$ value is defined as the probability of obtaining a certain value for the coefficient in the case of null hypothesis of no correlation.

The values in Table 2 show that the bar indicators used in different works but using similar criteria to define the bar are in good agreement. The values obtained from the correlation tests are not close to the unity, nevertheless, this fact does not indicate that the correlations are not significant. As the values compared come from different bar indicators and are estimated using completely different methodology, a tight linear correlation between them is not to be expected. The values of these rank correlations are in all cases significant, i.e. they are significantly different from the zero value as is indicated by the low $p$ values. The agreement is better for the cases where the values of the bar strength came from a similar methodology as is the case in Block et al. (2001), Buta et al. (2004), and Speltincx et al. (2008). Our bar indicators have significant positive correlations with these indicators, except for the case of the values quoted in Block et al. (2001). The correlation between the values of our bar indicators and the values from these authors, while given positive correlations, cannot be considered significant at the confidence level of $95 \%$. Likewise, our values are
Table 2. Comparison of the correlation coefficients between the different bar indicators for the galaxies in common.

\begin{tabular}{|c|c|c|c|c|c|c|c|}
\hline & $B_{M}$ & $B_{P}$ & Block & Buta & Spelt. & Mari. & Whyte \\
\hline \multirow{4}{*}{$B_{M}$} & & 329 & 81 & 98 & 133 & 110 & 130 \\
\hline & & 0.99 & 0.12 & 0.34 & 0.37 & 0.12 & 0.45 \\
\hline & & 0.99 & 0.17 & 0.25 & 0.24 & 0.08 & 0.45 \\
\hline & & 0.92 & 0.12 & 0.35 & 0.36 & 0.11 & 0.32 \\
\hline \multirow{4}{*}{$B_{P}$} & & & 81 & 98 & 133 & 110 & 130 \\
\hline & 0.00 & & 0.15 & 0.36 & 0.39 & 0.18 & 0.48 \\
\hline & 0.00 & & 0.20 & 0.36 & 0.37 & 0.15 & 0.47 \\
\hline & 0.00 & & 0.15 & 0.25 & 0.26 & 0.09 & 0.34 \\
\hline \multirow{4}{*}{ Block } & & & & 75 & 52 & 46 & 66 \\
\hline & 0.28 & 0.18 & & 0.83 & 0.79 & 0.66 & 0.75 \\
\hline & 0.09 & 0.05 & & 0.79 & 0.56 & 0.69 & 0.74 \\
\hline & 0.11 & 0.07 & & 0.69 & 0.72 & 0.54 & 0.57 \\
\hline \multirow{4}{*}{ Buta } & & & & & 68 & 63 & 67 \\
\hline & 0.00 & 0.00 & 0.00 & & 0.80 & 0.60 & 0.82 \\
\hline & 0.00 & 0.00 & 0.00 & & 0.76 & 0.59 & 0.80 \\
\hline & 0.00 & 0.00 & 0.00 & & 0.58 & 0.44 & 0.61 \\
\hline \multirow{4}{*}{ Spelt } & & & & & & 106 & 84 \\
\hline & 0.00 & 0.00 & 0.00 & 0.00 & & 0.47 & 0.62 \\
\hline & 0.00 & 0.00 & 0.00 & 0.00 & & 0.55 & 0.70 \\
\hline & 0.00 & 0.00 & 0.00 & 0.00 & & 0.40 & 0.53 \\
\hline \multirow{4}{*}{ Mari. } & & & & & & & 73 \\
\hline & 0.21 & 0.06 & 0.00 & 0.00 & 0.00 & & 0.61 \\
\hline & 0.26 & 0.12 & 0.00 & 0.00 & 0.00 & & 0.60 \\
\hline & 0.26 & 0.12 & 0.00 & 0.00 & 0.00 & & 0.46 \\
\hline \multirow[t]{3}{*}{ Whyte } & 0.00 & 0.00 & 0.00 & 0.00 & 0.00 & 0.00 & \\
\hline & 0.00 & 0.00 & 0.00 & 0.00 & 0.00 & 0.00 & \\
\hline & 0.00 & 0.00 & 0.00 & 0.00 & 0.00 & 0.00 & \\
\hline
\end{tabular}

Notes. In the upper diagonal we find the number of galaxies in common and the values of the Pearson, Spearman, and Kendall correlation coefficients. In the lower diagonal we find the respective $p$ values of the correlations. Values of 0.00 indicate $p$ values lower than $10^{-5}$.

not well correlated with the ellipticities of the bar as quoted in Marinova \& Jogee (2007). This is not surprising, we have seen in Sect. 4 that our method is sensitive to the bar shape, in the sense that the rounder the bars, the lower the power in the $m=2$ spectrum. We have good agreement, however, with the $f_{\mathrm{bar}}$ indicator used by Whyte et al. (2002). We can conclude that there is, in general, a reasonable agreement between the different bar indicators used in the literature.

\section{Distribution of the bar indicators}

In this section we use the values of our bar indicators as a measure of the general morphologies of galaxies indicated in the RC3 catalogue. In this catalogue the galaxies have been divided visually in three populations, namely SA, SAB, and SB, according to the visual strength of the bar, if present. In a SA galaxy no bar is present as seen by visual inspection, SB galaxies show a clear bar, while SAB galaxies are a mixed class were we can find small bars, ovals, and unclear cases. Our bar indicators give a numerical value for the bar strength and we would like to check whether our indicators reproduce the general properties of 
Bar Modulus

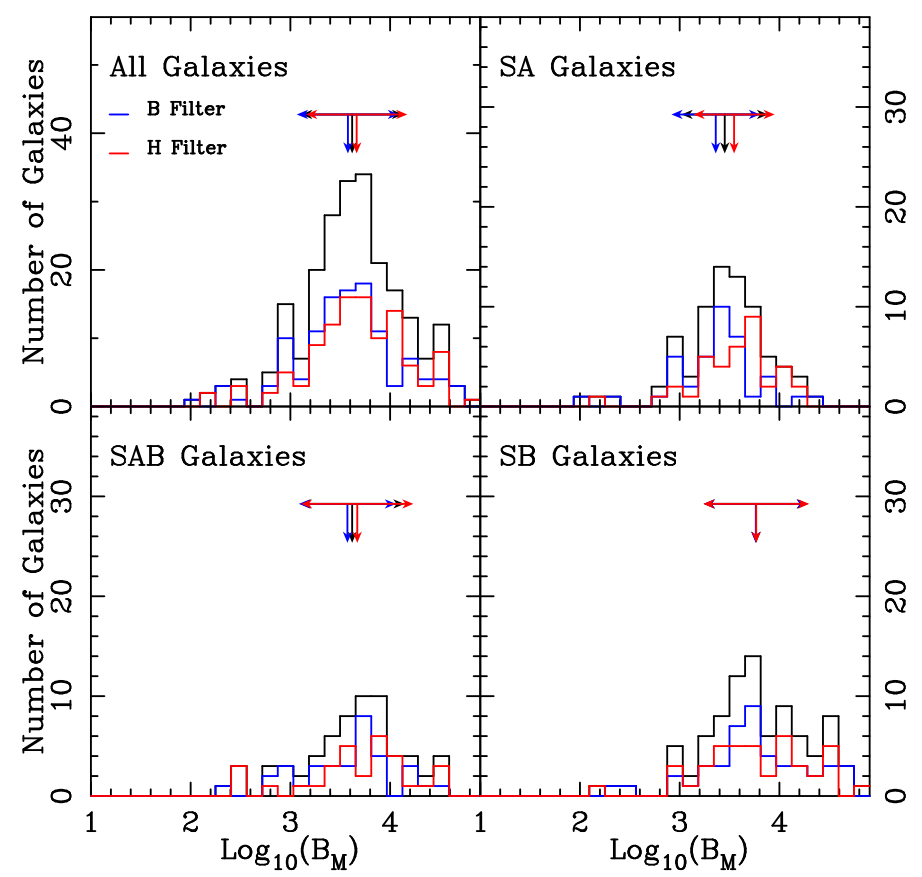

Fig. 13. Number of galaxies as a function of their bar modulus in the OSU sample. The upper left panel includes all galaxies, while the three others include only the SA, SAB, and SB families. In each panel the solid line corresponds to the values obtained for all the images irrespective of the filter used. We also separate the values for the galaxies studied using the $B$ filter (blue) and $H$ filter (red). The scales for the ordinate are not the same for all panels. The vertical arrows indicate the mean value for each population, while the length of the horizontal arrows indicate the value of the sigma of the distribution.

galaxies as derived from visual inspection. Our indicators should be able to recover the three galaxy populations in a general way. Thus, we can expect that barred galaxies from the SB type should have higher values of the bar indicators, SA galaxies should get lower values, while mixed class SAB should also produce a set of mixed values.

Figure 13 gives histograms of the number of galaxies as a function of their bar modulus in the OSU sample. The top left panel includes all the galaxies. The solid line corresponds to the distribution obtained using all the galaxy images, irrespective of the filter used. The distributions in blue correspond to the galaxy images obtained with the blue $B$ filter, while the distributions in red correspond to the galaxy images obtained with the infrared $H$ filter. We used the same codification for the other panels of the figure, where we separated the galaxies according to their classification in the RC3 catalogue in SA (non-barred), SAB (intermediate), or SB (barred) galaxies. The vertical arrows indicate the position of the mean of each population, while the horizontal arrows show the length of the standard deviation within each sample.

Using these distributions we can perform a series of statistical tests to ascertain if there are any differences between the galaxy populations. We start with the top left panel to see whether we can find any differences between the global population and blue or infrared populations. For this comparison, we can use measures of location for the distribution of the parameters. Here we use the standard mean and also robust measures of location, such as the median and $\alpha$-trimmed means, which are less biased by outliers than the classic standard mean. In the same way we can use the classic standard deviation as a measure of dispersion around the mean, but we can also use more robust measures of dispersion, such as the normalised standarised median absolute deviation (NMAD). The mean value of the global population is 3.6, while the standard deviation gives a value of 0.5 . Using only the blue or the infrared populations, the mean and standard deviation obtained are also 3.6 and 0.5. The median for the combined population also gives a value of 3.6, while the median values of the $B$ and $H$ populations are both of 3.7. The NMAD is computed to have a value of 0.4 in the three cases. In the case of the $\alpha$-trimmed means we also obtain values of 3.6 for the three populations. Thus, our data allow us to conclude that there are no differences in the bars detected with $B$ or $H$ passbands using the Fourier technique. This is in agreement with the results obtained in Sect. 5. This is confirmed by statistical tests on the mean difference of the location measures for the three distributions. An standard $t$-test on the difference of means, assuming that the populations are normal is unable to detect any difference in the means of the three galaxy distributions. This is true also for the case of the non-parametric statistical tests on the difference of the median values as the Wilcoxon-Mann-Whitney test or the robust generalisation of this test proposed by Mee (1990). A global non-parametric test for the comparison of the distributions, such as the Kolmogorov-Smirnov test, is also unable to separate the entire population from the population in the $B$ or $H$ passbands at the $95 \%$ confidence level.

If we repeat our analysis but this time separate the three galaxies populations using their SA, SAB, and SB visual class, we obtain similar results. None of the robust tests are able to obtain any difference between the combined population and the population obtained from the $B$ or $H$ passbands. Thus, all the distributions can be considered equal from a statistical point of view. We can find no differences in the bar detection levels using the blue or infrared passbands with our technique. Contrary to the methods used by other authors (Laurikainen \& Salo 2002; Marinova \& Jogee 2007), our method seems to be able to detect bars irrespective of the filter used to obtain the galaxy image.

We can also compare the distribution of the values of the bar indicator $B_{M}$ separately for the SA, SAB, and SB galaxies also using the standard and robust measures of location. For the SA population, the mean, median, and $\alpha$-trimmed mean give values of 3.46, 3.51, and 3.51, respectively. In the case of SAB galaxies, these values are 3.59, 3.64 and 3.62, respectively, while in the case of SB galaxies, we obtain $3.74,3.72$, and 3.73 . The differences between the SA, SAB, and SB are statistically significant at the $95 \%$ confidence level. The standard t-Student test on the difference of means give a confidence interval at the $95 \%$ level for the mean difference between SA and SB galaxies of $[-0.39,-0.15]$, excluding the zero value. This test is barely able to separate $\mathrm{SA}$ from $\mathrm{SAB}$ galaxies, giving a confidence interval of $[-0.23,0.00]$, while it is able to separate the SAB from the SB population with a confidence interval of $[-0.27,-0.03]$. This could indicate that the SAB population is more similar to the SA than to the SB population, but confirms the idea that this galaxy type is a mixed bag of values. The robust tests on the mean differences agree with the classical test. Thus, the generalised robust Wilcoxon-Mann-Whitney test from Mee (1990) is also able to separate the SA from the SB populations, giving a confidence interval at the $95 \%$ level on the ratio of means of $[0.59,0.72]$, excluding the 0.5 value. This test is also able to separate SA from the SAB populations with a confidence interval of $[0.52,0.64]$ and the SAB from the SB galaxies with confidence level of $[0.51,0.65]$. Thus our bar indicator $B_{M}$ performs very well in detecting the bar components of galaxies and 
Bar Power

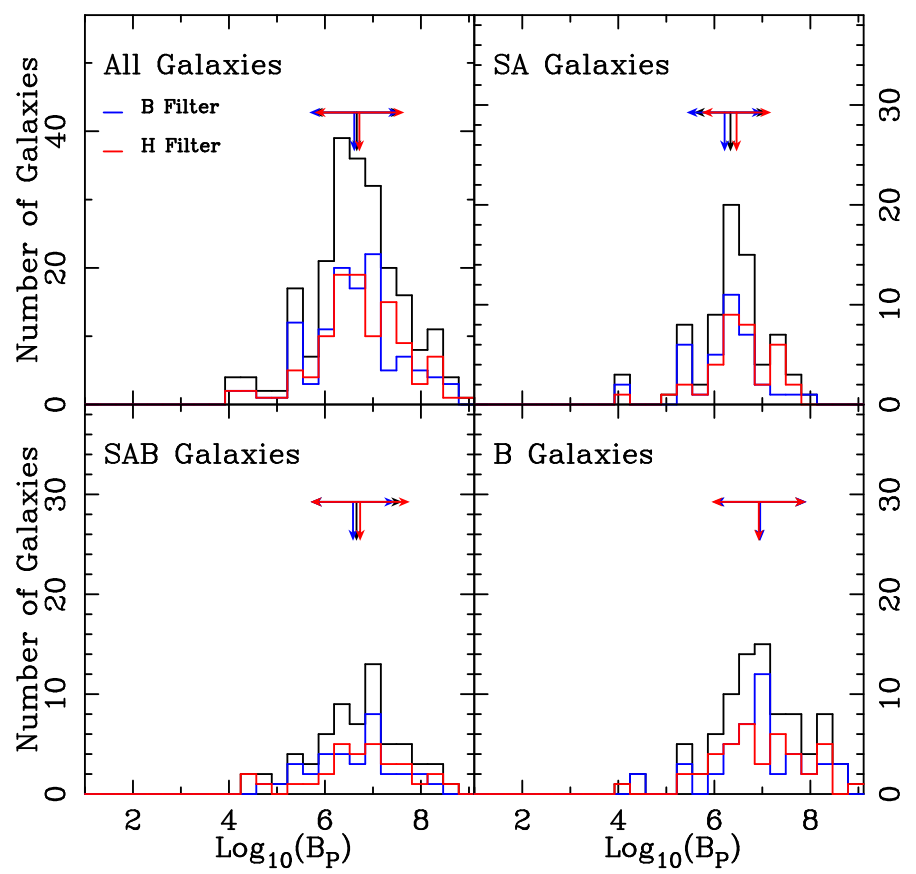

Fig. 14. Number of galaxies as a function of their bar power. The upper left panel includes all galaxies, while the three others are indicated separately for the SA, SAB, and SB families, respectively. The layout and symbols are as in Fig. 13.

is in overall good agreement with the visual classification of the RC3 catalogue from de Vaucouleurs et al. (1991). Similar results were found when using the bar power indicator instead of the bar modulus and are not given here for the sake of conciseness. The distributions and means obtained using this bar indicator are shown in Fig. 14, which shows the same layout as Fig. 13.

When we look at the distribution of our bar indicators, we can conclude that the relative strength of the bar in disc galaxies is a continuous parameter that is sometimes difficult to detect visually or with any qualitative measure. It is better to define the strength of the bar with a quantitative measure as the $B_{M}$ or $B_{p}$ parameters obtained from the Fourier analysis of the images. The values of this parameter in normalised images span about three orders of magnitude and could be used to define the degree of the bar phenomenon in a particular galaxy. Using the values from our analysis, we can define the set of non-barred galaxies as the galaxies with $\log _{10}\left(B_{M}\right)<3$, the set of mildly barred galaxies as galaxies with $3<\log _{10}\left(B_{M}\right)<3.5$, and the set of barred galaxies as those with $\log _{10}\left(B_{M}\right)>3.5$. This quantitative classification could be particularly useful when studying galaxies with no previous qualitative classification as any set of galaxies that is not included in the RC3 catalogue with cosmological interest

We can try also to use the normalised bar indicators to see if these features can be enhanced. In Fig. 15 we show the distribution of the values of the natural logarithm of the normalised bar indicator $B_{M 0}$, where we normalised the bar component modulus to the modulus of the $m=0$ spectrum, which corresponds to the strength of the underlying disc. The layout of Fig. 15 is also similar also to that in Fig. 13. As for the former cases, the mean value of the total distribution cannot be distinguished from the mean value of the distribution of $B$ or $H$ passbands. Moreover, as in the case of the $B_{M}$ indicator, the total distribution, the distribution of $B$ passband images, and the distribution of $H$ passband images cannot be differentiated from a statistical point of view.
Normalized Bar modulus $\mathrm{B}_{\mathrm{MO}}$

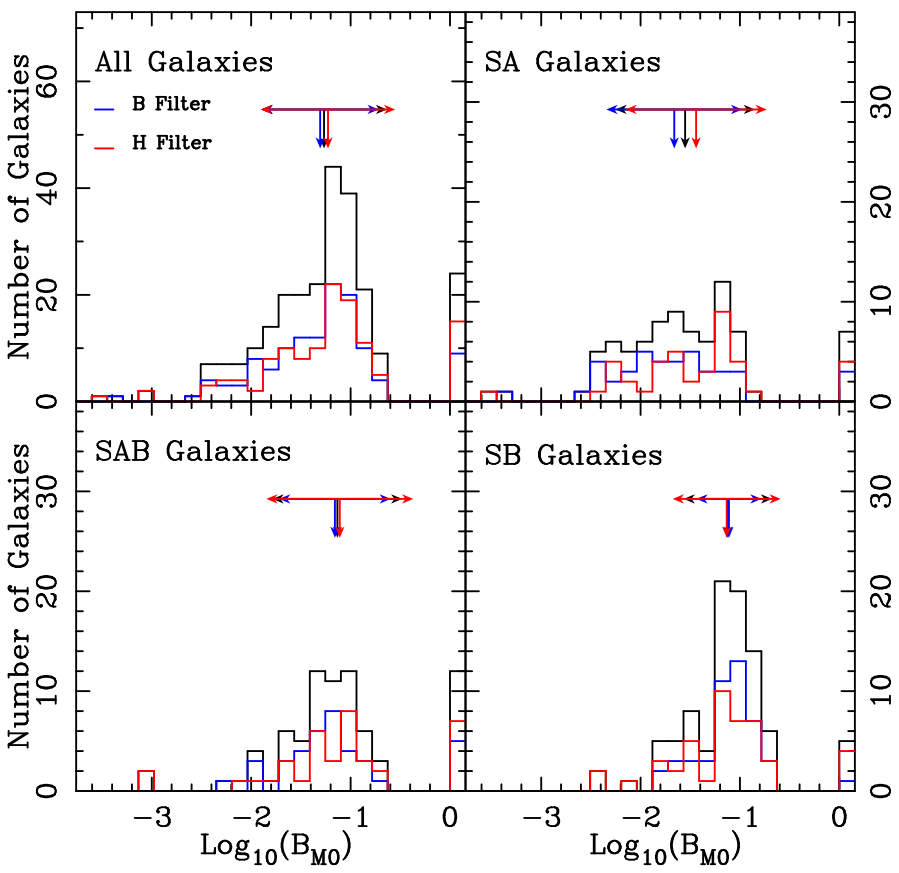

Fig. 15. Number of galaxies as a function of the natural logarithm of the $B_{M 0}$ bar indicator. The upper left panel includes all galaxies, while the three others are indicated separately for the SA, SAB, and SB families, respectively. The layout and symbols are as in Fig. 13.

As in the case of the non-normalised indicators the only significant effect is found when analysing the difference between the population of SA, SAB, and SB galaxies. The $95 \%$ confidence interval for the mean difference between SA and SB galaxies in a standard t-Student test is of $[-0.61,-0.37]$, clearly excluding the zero value and indicating a significant difference between SA and SB galaxies. This is true also for SA and SAB galaxies with a confidence interval of $[-0.38,-0.13]$ and for $\mathrm{SAB}$ and SB galaxies, with confidence interval $[-0.35,-0.11]$. The robust generalisation of the Wilcoxon-Mann-Whitney test performs equally well, clearly separating the three galaxy types. The normalised values seem to perform a bit better when trying to separate the galaxy types, but we must stress that all the differences between galaxy types result to be statistically significative despite any of our bar indicators used. If for instance, we use the normalisation by the sum of all the moments, i.e. the bar indicator $B_{M \sum}$, the results are again similar to the results obtained with any of the non-normalised indicators. The distributions for the values of the natural logarithm of $B_{M}$ are shown in Fig. 16. A similar result is obtained using the $B_{M 2}$ indicator; see Fig. 17.

\section{Discussion and conclusions}

In this paper we have introduced a new method to study the spiral structure of disc galaxies. This method uses a two-dimensional FFT on galaxy images. It is based on a similar method initially proposed by Considère \& Athanassoula (1982) for the HII region distribution and, independently, by Iye et al. (1982) who used this method on a photometric image of the galaxy NGC 4254. Later, Considère \& Athanassoula (1988) used it on a sample of images of bright spiral galaxies. These authors considered only the maximum component of each spectrum but the galaxy spectra can be very complicated owing to the presence of various components of different multiplicities. The method 
Normalized Bar Modulus $\mathrm{B}_{\Sigma \mathrm{M}}$

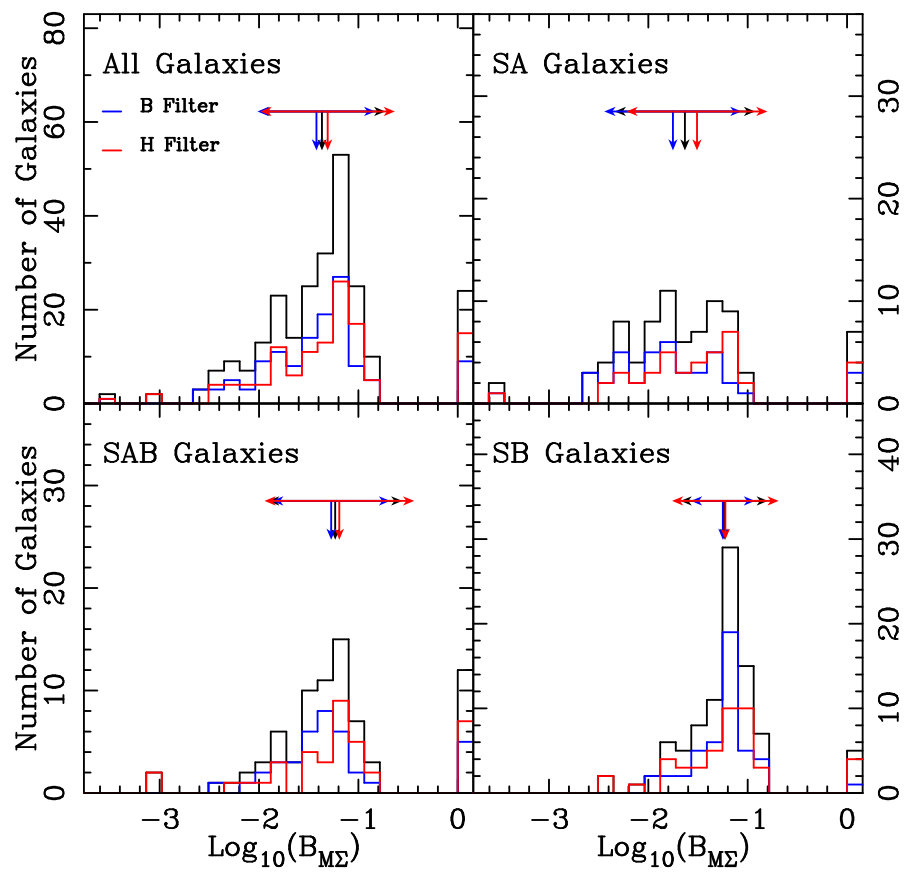

Fig. 16. Number of galaxies as a function of the natural logarithm of the $B_{M \sum m}$ bar indicator. The upper left panel includes all galaxies, while the three others are indicated separately for the SA, SAB, and SB families, respectively. The layout and symbols are as in Fig. 13.

was fully developed by García-Gómez \& Athanassoula (1991) for the HII region distribution. These authors introduced the possibility of dealing with the whole complexity of the Fourier spectra, separating all the signals present in each spectrum. In this paper we adapted this method to be applied on photometric galaxy images. Some other authors also applied the two-dimensional FFT on photometric galaxy images, for instance, Block \& Puerari (1999), Block et al. (2001), and Buta et al. (2003, 2004). In these papers however, only the maximum component was used for the analysis of the spiral structure. In a later paper, Buta et al. (2005) made an effort to include more components for each spectrum in a way similar to the present method. Our method is more general, however, as we are free to use as many components as necessary to obtain very good fits of the galaxy spectra, while we can recover the whole spiral structure of any galaxy image with great accuracy.

We used this method extensively on two large homogeneous galaxy samples, namely the Frei sample, Frei et al. (1996) and the Ohio State University Bright Spiral Galaxy Survey (OSU) Eskridge et al. (2002). In this particular paper, we only concentrated on the detection and analysis of barred structures. We introduced some bar strength indicators based on the Fourier decomposition of the galaxy images. These new bar indicators perform equally well from a statistical point of view as other bar indicators, namely the bar torque introduced by Buta \& Block (2001) and Laurikainen \& Salo (2002) and the bar strength measured by the $f_{\text {bar }}$ introduced by Abraham \& Merrifield (2000) and used by Whyte et al. (2002). Our bar indicators are not so well correlated with the bar ellipticities, as suggested by Athanassoula (1992), which were used in Marinova \& Jogee (2007).

The first point to be stressed is that we are able to detect the barred component with comparable strength in any of the galaxy passbands used to obtain the galaxy images in the former
Normalized Bar Modulus $\mathrm{B}_{\mathrm{M} 2}$

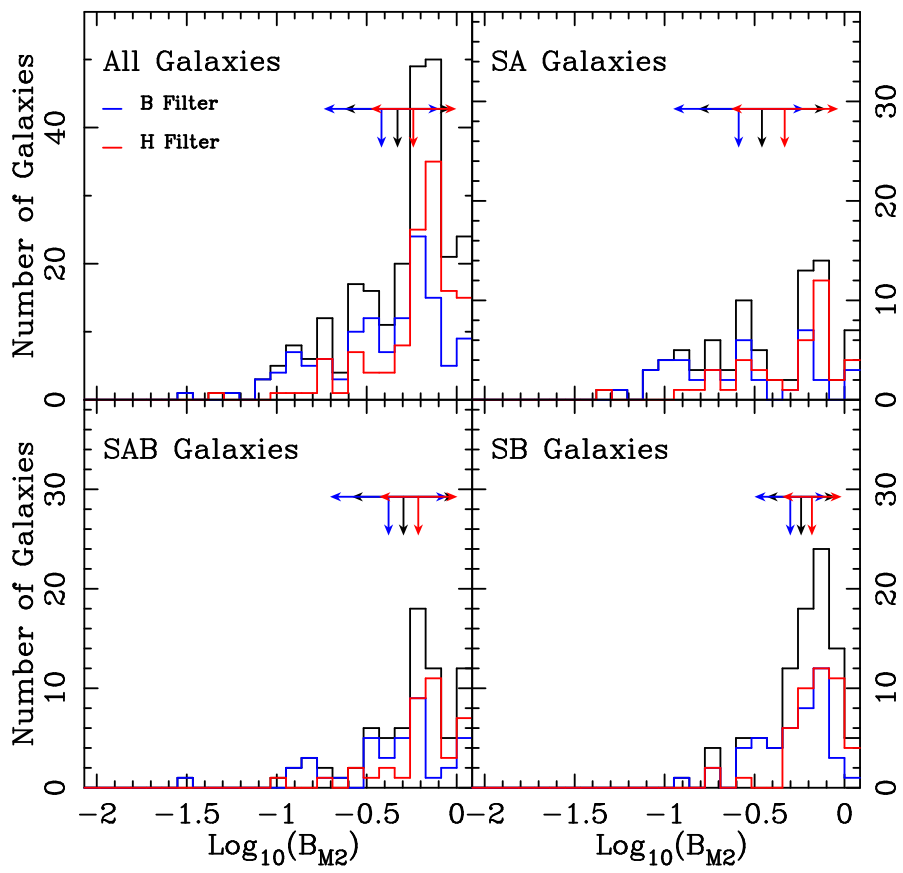

Fig. 17. Number of galaxies as a function of the natural logarithm of the $B_{M 2}$ bar indicator. The upper left panel includes all galaxies, while the three others are indicated separately for the SA, SAB, and SB families, respectively. The layout and symbols are as in Fig. 13.

samples. Thus, we do not find evidence of the case of a higher fraction of barred galaxies in the infrared as has been suggested by Eskridge et al. (2000) or Marinova \& Jogee (2007). The distribution of bar strength for the galaxy images obtained in the blue cannot be distinguished from the distribution of the bar strength obtained for the galaxy images obtained in the infrared region of the spectrum. When using our bar indicators in the Ohio sample, there is a minor effect that is more statistically significant for the case of stronger bars in the blue than for the galaxies in the far-infrared. On the other hand, our bar indicators are able to statistically separate the $\mathrm{SA}, \mathrm{SAB}$, and $\mathrm{SB}$ population of galaxies in order with the visual classification of the galaxies in the RC3 catalogue. Our method is able to separate the bar component from the rest of the spiral components and in about the $95 \%$ of the galaxies is able to detect a bar component, irrespective of its galaxy type. Thus, the vast majority of galaxies seem to harbour a bar in its stellar disc.

The strength of this bar, however, can be measured within a range of about three orders of magnitude. Thus the visual classification of galaxies as barred or non-barred is clearly justified. Our parameters could be used, however, as the basis of a more quantitative classification of the bar phenomenon.

This is intended to be the first paper of series in which we want to study the spiral structure of disc galaxies deeply. We intend to extend our analysis to the other spiral components with the aim of searching any links between the global spiral structure and the dynamical properties of galaxy discs. Another possible application of out method could be the study of the strength of the bar in a set of galaxies of cosmological interest. We would be able to study whether galaxies at larger distances appear to be more barred than neighbouring galaxies. This Fourier technique could be also used for large samples of galaxies. In this case, major issues should be addressed. The first should be of course the determination of the deprojection angles, 
as the Fourier technique should be used on a face on image of the galaxy. The deprojection methods used by different authors and using different methodologies can sometimes give very different results (Barberà et al. 2004; García-Gómez et al. 1991. Particularly for nearly face-on galaxies. For these cases, the PA could only be determined with some certainty using some information on the velocity field of the disc. For galaxies with inclination angles of values $40^{\circ}<$ IA $<65^{\circ}$ all the deprojection methods give comparable results and these galaxies could be deprojected using an automated procedure. A second important issue is to determine the parameter $r_{\min }$ used in our calculations, namely the size of the galaxy bulge. This value could be computed in a large sample using automated decomposition of the photometric galaxy profile. We have shown that the Fourier analysis is not very sensitive to this value as long as we clearly avoid the inner bulge. We think that this value could be computed with enough precision using the photometric profiles of the galaxies. The use of the Fourier analysis of the spiral structure in these large samples of cosmological interest could be very useful to understand the evolution of the spiral structure of galaxy discs.

Acknowledgements. We thank the anonymous referee for clear and acute comments, which have certainly improved the quality of this paper. We also thank David Block and Laura Whyte for providing us with the results of the $Q_{2}$ and $F_{\mathrm{b}}$ measurements, respectively. E.A. and A.B. acknowledge financial support to the DAGAL network from the People Programme (Marie Curie Actions) of the European Union's Seventh Framework Programme FP7/2007-2013/ under REA grant agreement number PITN-GA-2011-289313. We also wish to thank Ohio State University for the public release of the OSUBSGS galaxy sample.

\section{References}

Abraham, R. G., \& Merrifield, M. R. 2000, AJ, 120, 2835

Abraham, R. G., Merrifield, M. R., Ellis, R. S., Tanvir, N. R., \& Brinchmann, J. 1999, MNRAS, 308, 569

Aguerri, J. A. L. 1999, A\&A, 351, 43

Aguerri, J. A. L., Beckman, J. E., \& Prieto, M. 1998, AJ, 116, 2136

Aguerri, J. A. L., Muñoz-Tuñon, C., Varela, A. M., \& Prieto, M. 2000, A\&A, 361,841

Aguilar, L. A., \& Merritt, D. 1990, ApJ, 354, 33

Athanassoula, E. 1992, MNRAS, 259, 328

Athanassoula, E. 1996, in Barred Galaxies, eds. R. Buta, D. Crocker, \& B. Elmegreen, ASPC conf. Ser., 91, 309

Athanassoula, E. 2002, ApJ, 569, L83

Athanassoula, E. 2003, MNRAS, 341, 1179

Athanassoula, E. 2005a, MNRAS, 358, 1477

Athanassoula, E. 2005b, Celest. Mech. \& Dyn. Astron., 91, 9

Athanassoula, E., \& Misiriotis, A. 2002, MNRAS, 330, 35

Athanassoula, E., Morin, S., Wozniak, H., et al. 1990, MNRAS, 245, 130

Barberà, C., Athanassoula, E., \& García-Gómez, C. 2004, A\&A, 415, 849

Binney, J., \& Tremaine, S. 1987, Galactic Dynamics (Princeton University Press)

Block, D.L, \& Puerari, I. 1999, A\&A 342, 627

Block, D. L., Puerari, I., Knapen, J. H., et al. 2001, A\&A, 375, 761
Block, D. L., Bournaud, F., Combes, F., Puerari, I., \& Buta, R. 2002, A\&A, 394, L35

Block, D. L., Buta, R., Knapen, J. H., et al. 2004, AJ, 128, 183

Buta, R., \& Block, D. L. 2001, ApJ, 550, 243

Buta, R., Block, D. L., \& Knapen, J. H. 2003, AJ, 126, 1148

Buta, R., Laurikainen, E., \& Salo, H. 2004, AJ, 127, 279

Buta, R., Vasylyev, S., Salo, H., \& Laurikainen, E. 2005, AJ, 130, 506

Buta, R., Laurikainen, E., Salo, H., Block, D. L., \& Knapen, J. H. 2006, AJ, 132, 1859

Chapelon, S., Contini, T., \& Davoust, E. 1999, A\&A, 345, 81

Combes, F., \& Sanders, R. H. 1981, A\&A, 96, 164

Considère, S., \& Athanassoula, E. 1982, A\&A, 111, 82

Considère, S., \& Athanassoula, E. 1988, A\&AS, 76, 365

Danver, C. G. 1942, Ann. Obs. Lund, 10, 7

de Vaucouleurs, G. 1963, ApJS, 8, 31

de Vaucouleurs, G., de Vaucouleurs, A., Corwin, H. G., et al. 1991, Third Reference Catalogue of Bright Galaxies, RC3 (New York: Springer)

Durbala, A., Buta, R., Sulentic, J. W., \& Verdes-Montenegro, L. 2009, MNRAS, 397, 1756

Elmegreen, B. G., \& Elmegreen, D. M. 1985, ApJ, 288, 438

Eskridge, P. B., Frogel, J. A., Pogge, R. W., et al. 2000, AJ, 119, 536

Eskridge, P. B., Frogel, J. A., Pogge, R. W., et al. 2002, ApJS, 143, 73

Fisher, R. A. 1921, Metron, 1, 3

Frei, Z., Guhathakurta, P., Gunn, J., \& Tyson, J. A. 1996, AJ, 111, 174

García-Gómez, C., \& Athanassoula, E. 1991, A\&AS, 89, 159

García-Gómez, C., Barberà, C., Athanassoula, E., Bosma, A., \& Whyte, L. 2004, A\&A, 421, 595

Grosbol, P., Patsis, P. A., \& Pompei, E. 2004, A\&A, 423, 849

Heller, C., Shlosman, I., \& Athanassoula, E. 2007, ApJ, 657, L65

Huber, P. J. 1981, Robust Statistics (Wiley)

Iye, M., Okamura, S., Hamabe, M., \& Watanabe, M. 1982, ApJ, 256, 103

Kennicutt, R. C., Jr. 1981, AJ, 86, 1847

Knapen, J. H., Shlosman, I., \& Peletier, R. F. 2000, ApJ, 529, 93

Kormendy, J. 1979, ApJ, 227, 714

Laurikainen, E., \& Salo, H. 2002, MNRAS, 337, 1118

Laurikainen, E., Salo, H., Buta, R., \& Vasylyev, S. 2004, MNRAS, 355, 1251

Laurikainen, E., Salo, H., Buta, R., et al. 2006, AJ, 132, 1859

Ma, J. 2001, Chin. J. Astron. Astrophys., 1, 395

Marinova, I., \& Jogee, S. 2007, ApJ, 659, 1176

Martin, P. 1995, AJ, 109, 2428

Martinet, L., \& Friedli, D. 1997, A\&A, 323, 363

Mee, R. W. 1990, J. Am. Stat. Assoc., 85, 793

Menéndez-Delmestre, K., Sheth, K., Schinnerer, E., Jarrett, T. H., \& Scoville, N. Z. 2007, ApJ, 657, 790

Ohta, K. 1996, in Barred Galaxies, eds. R. Buta, D. A. Crocker, \& B. G. Elmegreen (San Francisco: ASP), ASP conf. Ser., 91, 37

Ohta, K., Hamabe, M., \& Wakamatsu, K. 1990, ApJ, 357, 71

Press, W. H., Teulkosky, S. A., Vetterling, W. T., \& Flannery, B. P. 1994, Numerical Recipes (Cambridge University Press)

Regan, M. W., \& Elmegreen, D. M. 1997, AJ, 114, 965

Rozas, M., Knapen, J. H., \& Beckman, J. E. 1998, MNRAS, 301, 631

Sandage, A., \& Tammann, G. A. 1981, A Revised Shapley-Ames Catalog of Galaxies (Washington: Carneige Inst.)

Seigar, M. S, \& James, P. A. 1998, MNRAS, 299, 672

Shlosman, I., Peletier, R. F., \& Knapen, J. H. 2000, ApJ, 535, L83

Speltincx, T., Laurikainen, E., \& Salo, H. 2008, MNRAS, 383, 317

Sygnet, J. F., Tagger, M., Athanassoula, E., \& Pellat, R. 1988, MNRAS, 232, 733

Tagger, M., Sygnet, J. F., Athanassoula, E., \& Pellat, R. 1987, ApJ, 318, L43

Whyte, L. F., Abraham, R. G., Merrifield, M. R., et al. 2002, MNRAS, 336, 1281 